\title{
The Down syndrome critical region gene 1 short variant promoters direct vascular bed-specific gene expression during inflammation in mice
}

\author{
Takashi Minami,1,2 Kiichiro Yano,, ${ }^{3}$ Mai Miura, ${ }^{1}$ Mika Kobayashi,1 Jun-ichi Suehiro,, Patrick C. Reid, ${ }^{4}$
} Takao Hamakubo, ${ }^{1}$ Sandra Ryeom, ${ }^{5}$ William C. Aird, ${ }^{3}$ and Tatsuhiko Kodama ${ }^{1,2}$

\begin{abstract}
${ }^{1}$ Research Center for Advanced Science and Technology and ${ }^{2}$ Translational Systems Biology and Medicine Initiative (TSBMI), University of Tokyo, Tokyo, Japan.
${ }^{3}$ Department of Medicine, Center for Vascular Biology Research, Beth Israel Deaconess Medical Center and Harvard Medical School, Boston, Massachusetts, USA. 'PeptiDream Inc., Tokyo, Japan. ${ }^{5}$ Vascular Biology Program, Department of Surgery, Children's Hospital Boston,
\end{abstract} Boston, Massachusetts, USA.

\begin{abstract}
Down syndrome critical region gene 1 (DSCR-1) short variant (DSCR-1s) is an inhibitor of calcineurin/NFAT signaling encoded by exons 4-7 of DSCR1. We previously reported that VEGF induces DSCR-1s expression in endothelial cells, which in turn negatively feeds back to attenuate endothelial cell activation. Here, in order to characterize the role of the promoter that drives DSCR-1s expression in mediating inducible expression in vivo and to determine the functional relevance of DSCR-1s in inflammation, we targeted a DNA construct containing $1.7 \mathrm{~kb}$ of the human DSCR1s promoter coupled to the lacZ reporter to the hypoxanthine guanine phosphoribosyl transferase ( $\mathrm{Hprt}$ ) locus of mice. We determined that lac $Z$ was uniformly expressed in the endothelium of transgenic embryos but was markedly downregulated postnatally. Systemic administration of VEGF or LPS in adult mice resulted in cyclosporine A-sensitive reactivation of the DSCR1s promoter and endogenous gene expression in a subset of organs, including the heart and brain. The DSCR1s promoter was similarly induced in the endothelium of tumor xenografts. In a mouse model of endotoxemia, DSCR-1s-deficient mice demonstrated increased sepsis mortality, whereas adenovirus-mediated DSCR-1s overexpression protected against LPS-induced lethality. Collectively, these data suggest that the DSCR1s promoter directs vascular bed-specific expression in activated endothelium and that DSCR-1s serves to dampen the host response to infection.
\end{abstract}

\section{Introduction}

Endothelial cell phenotypes vary in structure and function, in space and time, and in health and disease (reviewed in refs. 1, 2 ). Endothelial cells are adapted to meet the diverse needs of the underlying tissue. Phenotypic heterogeneity is governed by a combination of extracellular signals and epigenetic forces (reviewed in ref. 3). Under normal conditions, signals from the extracellular environment are integrated at the transcriptional and posttranscriptional levels, resulting in tight control of hemostatic balance, leukocyte trafficking, barrier function, migration, and proliferation. When signaling is excessive, oversustained and/or spatially and temporally misplaced, pathology may ensue. Among the important regulatory mechanisms in the endothelium is negative feedback inhibition of transcription networks.

The Down syndrome critical region gene 1 (DSCR1) (also known as MCIP-1, calcipressin-1, Adapt 78, and RCAN), so designated because it resides within the Down syndrome critical region of human chromosome 21 , encodes a protein that binds to and

Authorship note: William C. Aird and Tatsuhiko Kodama contributed equally to this work.

Conflict of interest: The authors have declared that no conflict of interest exists.

Nonstandard abbreviations used: CsA, cyclosporine A; DSCR-1, Down syndrome critical region gene 1; DSCR-1L, DSCR-1 long variant; DSCR-1s, DSCR-1 short variant; HMVEC, human microvascular endothelial cell; Hprt, hypoxanthine guanine phosphoribosyl transferase; LLC, Lewis lung carcinoma.

Citation for this article: J. Clin. Invest. 119:2257-2270 (2009). doi:10.1172/JCI35738 inhibits the catalytic subunit of calcineurin (4-6). The DSCR1 gene includes 7 exons and 6 introns and encodes 4 different isoforms. The two major isoforms are referred to as DSCR-1 long variant (DSCR-1L) and DSCR-1 short variant (DSCR-1s). DSCR-1L contains exons $1,5,6$, and 7, whereas DSCR-1s contains exons 4, 5, 6 , and 7 . The two isoforms are differentially regulated; the $5^{\prime}$ promoter drives Notch/Hes-1-inhibitable expression of DSCR-1L (7), while an intergenic promoter located between exons 3 and 4 mediates calcineurin/NFAT-dependent expression of DSCR-1s (8).

We recently reported that VEGF and thrombin result in dramatic and rapid upregulation of DSCR-1s (8). VEGF-mediated induction of DSCR-1s was shown to involve the cooperative binding of NFATc and GATA2/3 to neighboring consensus motifs in the upstream promoter. DSCR-1s, in turn, feeds back to inhibit calcium/calcineurin/NFAT signaling, resulting in attenuation of inflammation and angiogenesis (8).

Here, we wished to extend our studies to the in vivo setting. We asked whether the DSCR1s promoter, as delineated in our cell culture studies, contains information for inducible expression in vivo. Moreover, we tested the hypothesis that DSCR-1-mediated attenuation of inflammation influences the host innate immune response. Here, we show that the DSCR1s promoter directs widespread basal expression of lacZ throughout the vascular tree in the developing embryo, while reporter gene activity is significantly downregulated in the postnatal period. However, in the adult, the DSCR1s promoter is upregulated in different vascular beds in 
A Hprt-targeting vector (pMpll8)

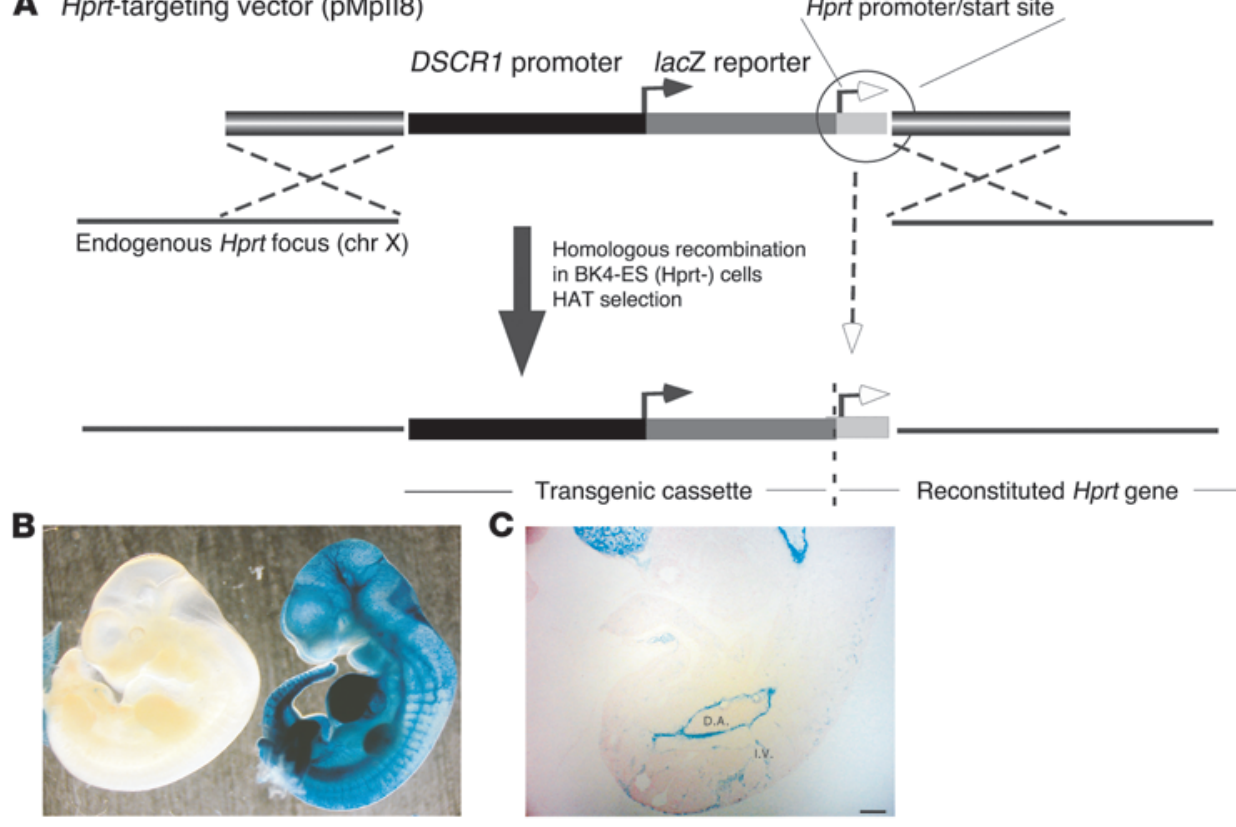

\section{Figure 1}

Generation of DSCR1s promoter containing, Hprt locus-targeted mice. (A) Schematic representation of the DSCR-1-lacZ construct and Hprt locus targeting. (B) Whole-mount lacZ staining of E11 Hprt-targeted embryos. Right: DSCR-1-lacZ E11 transgenic embryo. Left: Littermate control lacking the DSCR-1s-lacZ transgene. The two embryos were harvested and processed for lacZ staining in parallel. The results are representative of more than 30 embryos. (C) Representative lacZ staining from vertical sections of E11 transgenic embryo. I.V., intersomite vessel; D.A., dorsal aorta. Scale bar: $100 \mu \mathrm{m}$. response to VEGF and LPS and as a consequence of tumor growth. In addition, mice with a targeted deletion of both DSCR-1 isoforms demonstrated increased sepsis mortality, while overexpression of DSCR-1s protected against LPS-induced lethality. Taken together, the results provide new insights into vascular bed-specific expression of DSCR-1s in vivo and point to DSCR-1s as a potential therapeutic target in vascular inflammation.

\section{Results}

Hprt-targeted DSCR-1 intergenic promoter directs age-dependent expression in the intact endothelium. In a previous study, we demonstrated that VEGF and thrombin induced the expression of DSCR-1s in HUVECs, resulting in secondary attenuation of calcium/NFAT signaling (8). A region of the DSCR1s promoter between $-1,664$ and +83 was shown to contain information for VEGF and thrombin responsiveness (8). To determine whether the same promoter region directed inducible expression in vivo, we coupled the $-1,664 /+83$ DSCR1s promoter to the lacZ reporter gene and targeted the resulting transgenic cassette (DSCR-1-lacZ) to the Hprt locus of mice using homologous recombination (Figure 1A). Four independent high-percentage male chimeras, derived from 2 recombinant ES cell clones, were bred with wild-type mice to achieve germline transmission. $\mathrm{F}_{1}$ agouti females were mated with DSCR-1-lacZ-Hprt hemizygous males and harvested for embryos at various gestational ages. At E11, whole-mount lacZ staining revealed widespread expression of the transgene in the vasculature (Figure 1B). In cryosections, strong staining was observed in the dorsal aorta, intersomitic vessels, carotid arteries, caudal veins, the primary head vein branch, and the endocardium (Figure $1 \mathrm{C}$ shows dorsal aorta and intersomitic vessels). lacZ colocalized with endothelial PECAM-1 (data not shown). At E14, lacZ expression was markedly downregulated (data not shown). At E19, reporter gene activity was further reduced, with detectable expression limited to the endocardium, umbilical artery, and umbilical vein (data not shown). In adult mice, DSCR-1s-lacZ activity was detected in only a subset of endothelial cells in the brain, heart, lung, and kidney (Supplemental Figure 1, A-D; sup- plemental material available online with this article; doi:10.1172/ JCI35738DS1). Expression was also observed in occasional neurons, vascular smooth muscle cells, cardiomyocytes, and renal epithelial cells. In contrast, DSCR-1s-lacZ activity was undetectable in the liver, spleen, thigh skeletal muscle, and thymus (Supplemental Figure 1, E and F, shows liver and skeletal muscle, respectively). These findings suggest that the DSCR1s promoter, though widely expressed in the endothelium of E11 embryos, is downregulated in later stages of development and in adults.

Hprt-targeted DSCR-1 intergenic promoter directs vascular bed-specific expression in response to VEGF and LPS. Next, we wished to determine whether the DSCR1s promoter confers response to inflammatory stimuli in vivo. To that end, DSCR-1s-lacZ mice were systemically administered VEGF (500 $\mu \mathrm{g} / \mathrm{kg}$, i.v.) or LPS (10 mg/kg, i.p.). Organs were harvested 6 hours later and stained for lacZ. In wholemount preparations, the X-gal reaction product was detectable in the brain and heart of untreated mice and was further upregulated by VEGF and LPS (Figure 2, A and B). In contrast, lacZ staining was not observed in skeletal muscle under any condition (Figure $2 \mathrm{C}$ shows control and LPS treatment). By comparison, mice in which the Flt1 promoter was targeted to the Hprt locus (Flt-1-lacZ) demonstrated $\beta$-galactosidase activity in skeletal muscle vasculature (Figure 2C). Flt1 promoter activity was not altered by treatment with LPS or VEGF, suggesting that the stimulatory effect of these agonists is specific to the DSCR1s promoter.

Tissue sections from the DSCR-1s-lacZ mice revealed inducible expression of the promoter in the aorta, brain, lung, and kidney but not in the spleen, liver, or thymus (Supplemental Figure 2, A-D, and data not shown). VEGF-mediated induction of the DSCR-1s transgene in the aorta was restricted to the endothelial lining, as evidenced by colocalization of lacZ and PECAM-1 (Supplemental Figure 2A). In contrast, LPS-mediated induction of the transgene occurred in both the endothelial and smooth muscle cell layers of the aorta (Supplemental Figure 2A). Both VEGF and LPS significantly enhanced reporter gene expression in the microvascular endothelium of the brain (Supplemental Figure 2B). In the lung, 
A Control

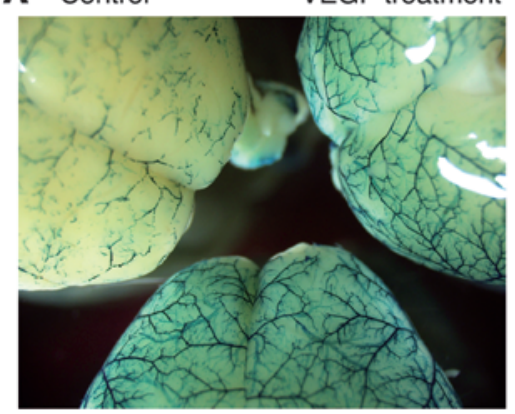

LPS treatment

C

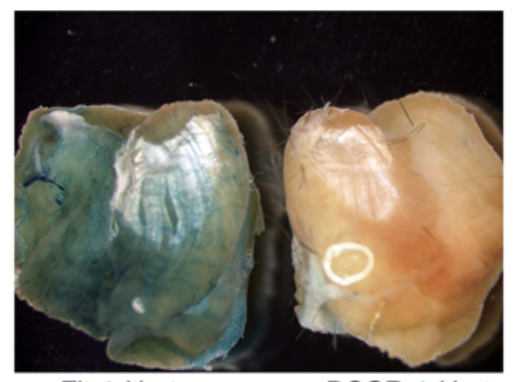

Flt-1-Hprt

VEGF treatment

\section{B Control}

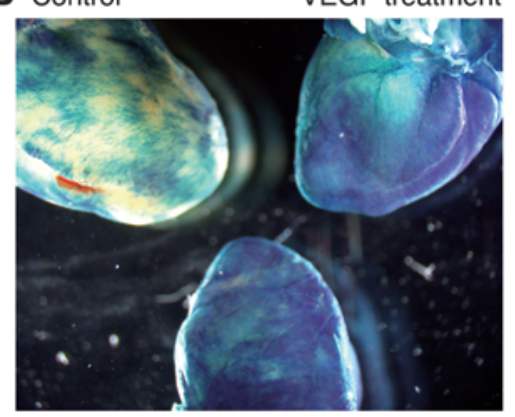

LPS treatment

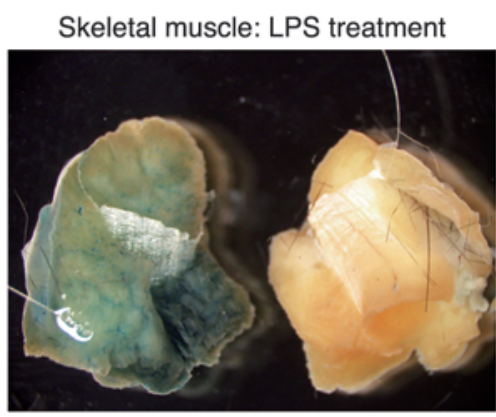

Flt-1-Hprt

\section{Figure 2}

Systemic administration of VEGF and LPS results in organ-specific increases in DSCR1s promoter activity. (A and B) Comparative whole-mount lacZ stains of brain (A) and heart (B) taken from untreated (control), LPS-treated, or VEGF-treated mice. (C) Wholemount lacZ stains of thigh skeletal muscle from Flt-1lacZ-Hprt or DSCR-1s-lacZ-Hprt mice treated without (left panel) or with LPS (right panel). systemic administration of VEGF and LPS resulted in increased transgene expression in both micro- and macrovascular endothelium (Supplemental Figure 2C). In the kidney, LPS administration increased lacZ expression in PECAM-1-negative cells of the glomerulus and proximal tubule, while VEGF administration induced expression mainly in glomerular endothelial cells (Supplemental Figure 2D). To determine whether expression of the transgene correlated with that of the endogenous gene, immunostaining was carried out using a mouse monoclonal anti-DSCR-1 antibody. VEGF stimulated DSCR-1 expression in the endothelial lining of multiple organs in a pattern that correlated with that of lacZ (Supplemental Figure 3 shows heart and kidney). Together, these data suggest that inflammatory mediators result in vascular bed-specific induction of DSCR1s promoter activity.

Real-time PCR analysis was used to quantify changes in transgene expression. Under basal conditions, lacZ mRNA expression was highest in the heart, followed by the brain, lung, and kidney (Figure 3A). Expression in skeletal muscle, spleen, and liver was below the level of detection. VEGF and LPS resulted in significant induction of lac Z transcripts in the heart (4.5-fold and 8.1-fold, respectively), brain (2.9-fold and 4.0-fold, respectively), lung (2.7-fold and 4.2-fold, respectively), and kidney (3.1-fold and 6.2 -fold, respectively), but not in spleen, liver, or skeletal muscle (Figure 3A). LPS-mediated induction of the endogenous Dscr1s gene was similarly restricted to the heart (25.3-fold), brain (7.0-fold), lung (10.3-fold), and kidney (9.3-fold) (Figure 3B). We have previously shown that i.v. administration of VEGF results in phosphorylation of Flk-1 in multiple organs, including those in which DSCR-1s is not induced (e.g., liver and skeletal muscle) (9). To confirm that this was the case in the current study, we carried out immunoprecipitation and Western blot analysis for phospho-Flk-1 in the lung, heart, skeletal muscle, liver, and spleen of control and VEGF-treated mice. Ten minutes after i.v. administration of VEGF, phosphorylation of Flk-1 was induced in the lung, heart, skeletal muscle, and liver, with comparable ratios of phospho-Flk-1 versus total Flk-1 (Supplemental Figure 4A). These results argue against organ-specific differences in Flk-1 signaling in mediating differential induction of DSCR-1s. Moreover, both VEGF and LPS induced expression of Egr-1 in all organs except the brain, indicating that these agonists result in widespread activation, even in DSCR-1snegative tissues (Supplemental Figure 4B). Finally, agonist treatment failed to alter DSCR-1L transcript levels (Figure 3B). Thus, VEGF and LPS promote vascular bed-specific expression of both the DSCR1s promoter and the endogenous Dscr1s gene.

Hprt-targeted DSCR-1 intergenic promoter directs expression in tumor endothelium. Solid tumors produce a variety of proangiogenic molecules and inflammatory cytokines, which have important paracrine effects on surrounding endothelial cells. Thus, we investigated whether the DSCR-1s transgene is activated in tumor blood vessels. To that end, B16-F1 melanoma and Lewis lung carcinoma (LLC) cells were implanted s.c. into the flank of DSCR-1s-lacZ-Hprt mice. When tumors reached approximately $2.5 \mathrm{~cm}^{3}$ in volume, the xenografts were harvested, sectioned, and stained for lacZ. As shown in Figure 4A, there was widespread reporter gene activity within both B16-F1 melanoma and LLC tumor neovessels. In double immunofluorescence studies, lacZ colocalized with endothelial PECAM-1 (Figure 4A, bottom). Consistent with these findings, endogenous DSCR-1 also colocalized with PECAM-1 in tumor vessels of both B16-F1 melanoma and LLC xenografts (Figure 4B).

Inducible expression of DSCR-1s in cultured microvascular endothelial cells is mediated by NFATc and GATA2. We previously demonstrated that VEGF and thrombin, but not TNF- $\alpha$, induced DSCR1s promoter activity in HUVECs (8). Given that systemic administration of LPS in mice induced expression of the Hprt-targeted DSCR-1 intergenic promoter and the endogenous Dscr1s gene in vivo, we wished to determine whether LPS directly activates the promoter 

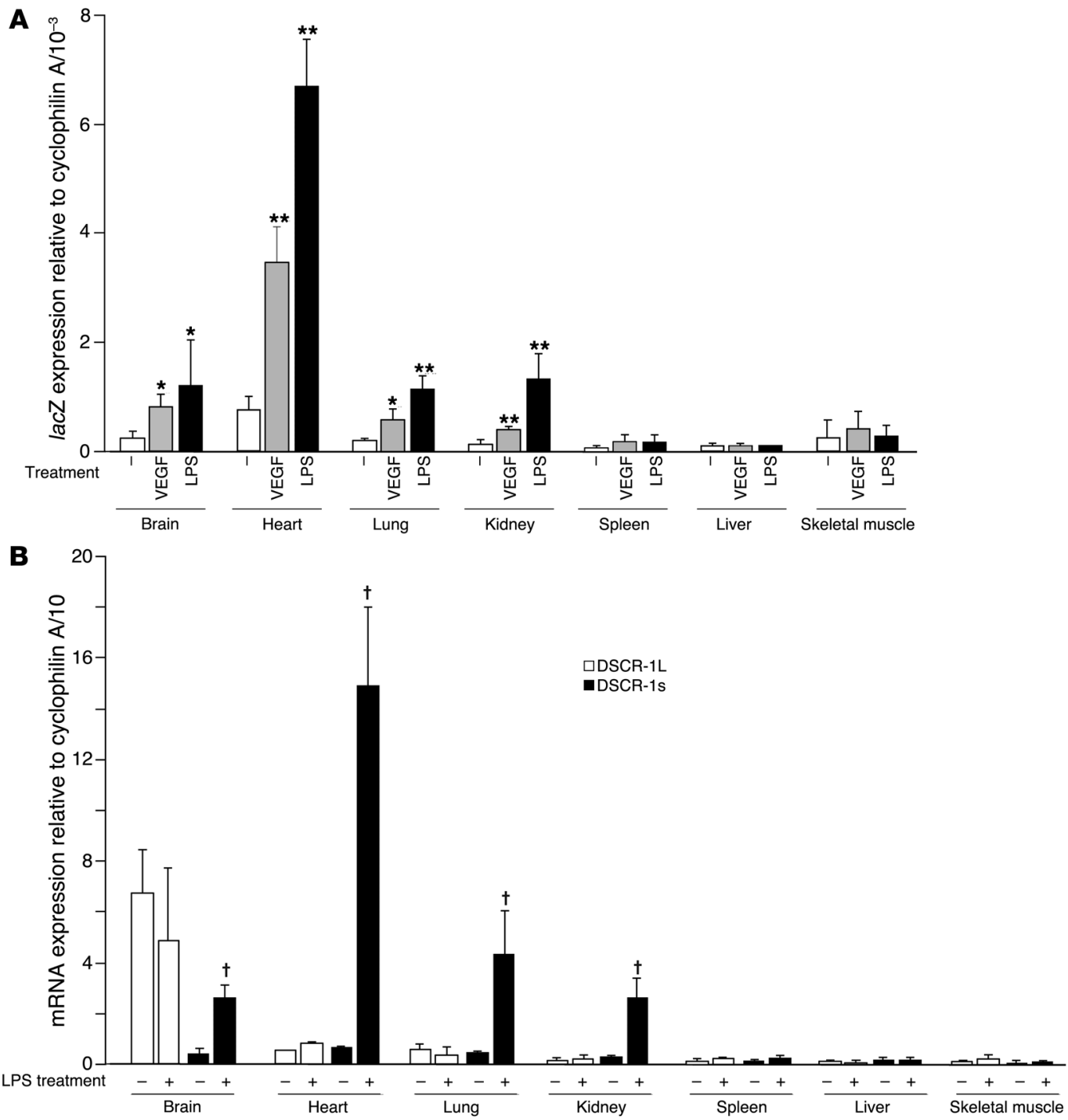

Figure 3

Systemic administration of VEGF and LPS results in organ-specific increases in lacZ and DSCR-1s mRNA expression. (A) Real-time PCR quantitation of lacZ expression levels in various organs from control mice, VEGF-treated mice, or LPS-treated mice. Data are expressed as mean \pm standard deviation; $n=5$ in each treatment. ${ }^{*} P<0.04,{ }^{* *} P<0.01$ compared with mock treatment in each organ. (B) Real-time PCR quantitation of DSCR-1L and DSCR-1s mRNA expression levels in various organs from LPS-treated mice. The results are the mean and standard deviation of expression levels relative to cyclophilin A, derived from at least 4 independent mice. ${ }^{\dagger} P<0.001$ compared with DSCR-1s expression levels without LPS treatment.

in endothelial cells. To that end, human microvascular endothelial cells (HMVECs) were transiently transfected with a plasmid containing $1.7 \mathrm{~kb}$ DSCR1s promoter coupled to luciferase (DSCR-1sluc). The transfected cells were treated in the absence or presence of various agonists. Consistent with our data in HUVECs, VEGF and thrombin stimulation of microvascular endothelial cells resulted in 11- and 8.2-fold induction of promoter activity, respectively, while TNF- $\alpha$ had only a modest effect on promoter expression (1.5-fold induction) (Figure 5A). In contrast, LPS failed to alter luciferase activity (Figure 5A). A similar pattern of response was observed for the endogenous Dscr1s gene (Figure 5B). These data suggest that the effect of LPS on DSCR-1s expression in vivo is mediated by secondary signals.
We reported that VEGF- and thrombin-mediated induction of DSCR-1 involves the cooperative binding of NFATc and GATA2 to neighboring consensus motifs in the upstream promoter (located at positions -220 and -254 , respectively) (4). GATA2, NFATc1, NFATc2, and/or NFATc3 are expressed in HMVECs (data not shown). To delineate which of these factors are responsible for mediating inducible expression of DSCR-1s, we employed siRNA to knock down their expression in HMVECs. The various siRNAs resulted in more than $75 \%$ downregulation of their respective target genes (Supplemental Figure 5). GATA2 knockdown significantly inhibited (63\%) VEGF-mediated induction of DSCR-1s mRNA, whereas single knockdown of NFATc1, NFATc2, or NFATc3 had no such effect (Figure 5C). In contrast, the VEGF 
A
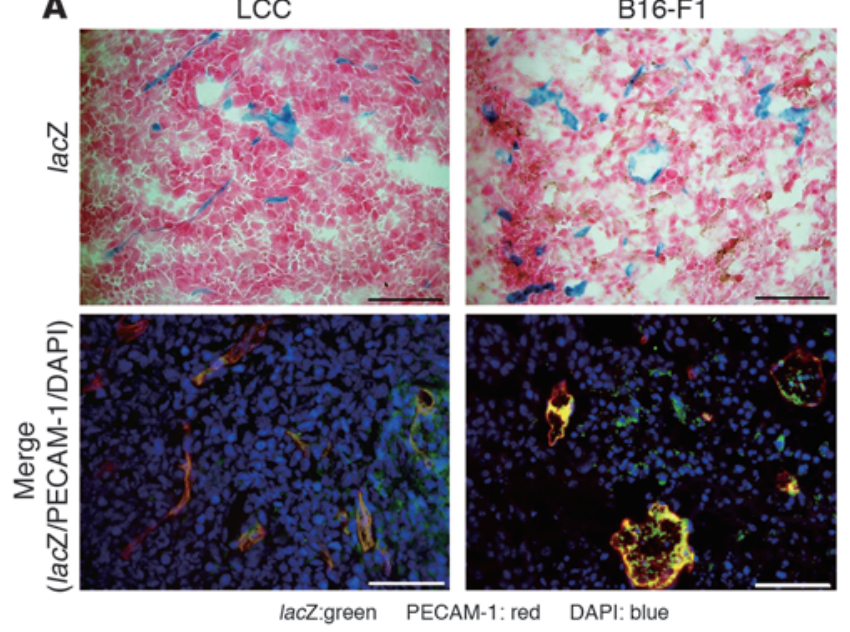

B
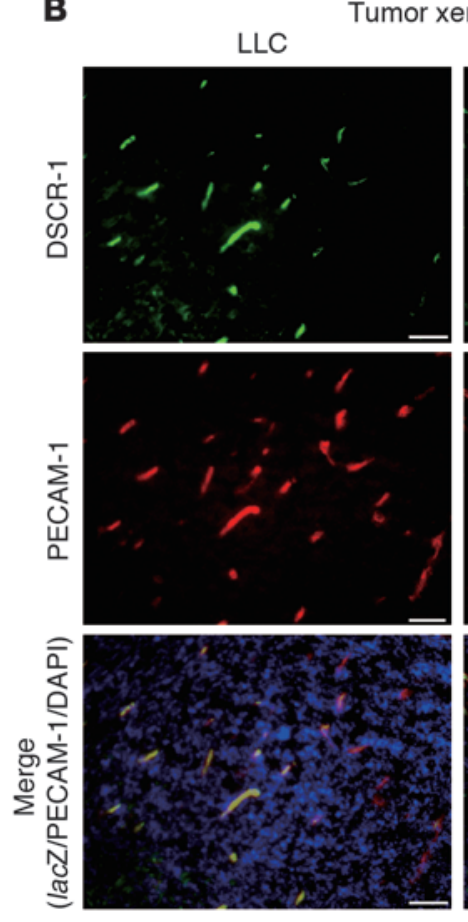

$\mathrm{B} 16-\mathrm{F} 1$

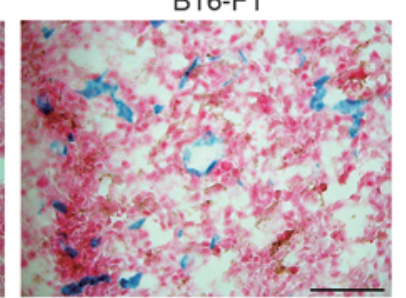

$\mathrm{B} 16-\mathrm{F} 1$
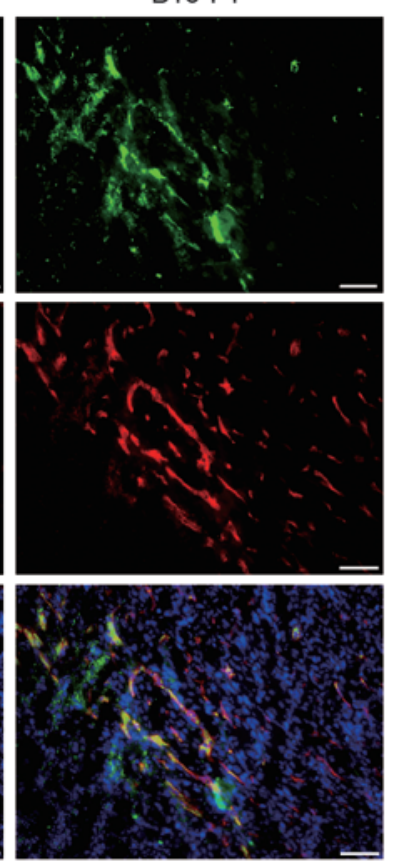

response was inhibited $32 \%$ by double knockdown of NFATc1 and NFATc $2 ; 52 \%$ by combined knockdown of NFATc1, NFATc2, and NFATc3; and 94\% by siRNA against NFATc1, NFATc2, and GATA2 (Figure 5C). Together, these data support an essential role for GATA2 and NFATc1, -c2, and -c3 transcription factors in mediating inducible DSCR-1s expression.

To investigate whether tumor-secreted factors induce DSCR1s promoter activity, we incubated DSCR-1s-luc-transfected HMVECs were incubated in the absence or presence of tumor cell-conditioned medium and assayed the cells 2 hours later for luciferase activity. As shown in Figure 6A, incubation of HMVECs with conditioned medium from LLC and B16-F1 melanoma cells resulted in a 7.2-fold and a 5.5-fold in DSCR1s promoter activation, respectively, while the medium from normal skin fibroblasts failed to upregulate the DSCR1s promoter. Cells residing in the inner core of solid tumors are exposed to a relatively hypoxic environment.

\section{Figure 4}

GATA- and NFATC-dependent DSCR1s promoter and protein expression is upregulated in tumor vasculature. (A) Upper panels: lacZ stainings were performed with 10- $u$ m cryosections of LLC and B16-F1 melanoma xenografts. Lower panels: Merged images of immunofluorescence staining with antibodies against lacZ (green) or PECAM-1 (red) or with DAPI (blue). lacZ/PECAM-1 double-positive cells are yellow/orange. Scale bars: $50 \mu \mathrm{m}$. (B) Cryosections from LLC and B16-F1 melanoma xenografts were stained with anti-DSCR-1 antibody (green), antiPECAM-1 antibody (red), and DAPI (blue). Merged images are shown in the lower panels. Scale bars: $50 \mu \mathrm{m}$.

Thus, we asked whether hypoxia induced DSCR1s promoter activity in transiently transfected HMVECs. As shown in Figure 6A, luciferase activity was not upregulated in cells grown in $2 \%$ oxygen for 24 hours. Promoter induction by tumor cell-conditioned medium was significantly inhibited by neutralizing anti-Flk-1 antibodies (Figure 6A). These data are consistent with a role for tumor-derived VEGF in mediating DSCR-1s expression. However, the inhibitory effect was not complete, suggesting a role for additional tumorderived mediators in this process. Finally, combined treatment with siGATA2 and cyclosporine A (CsA) completely blocked the effect of LLC-conditioned medium on DSCR1s promoter activation (Figure 6A). Thus, tumor cell-mediated induction of DSCR-1s involves GATA2- and NFATc-dependent pathways.

To confirm that endogenous DSCR-1s protein levels are similarly regulated by GATA2 and NFATc activity, we carried out Western blot analyses. As shown in Figure 6B, VEGF- or thrombin-mediated induction of DSCR-1s protein expression was profoundly inhibited by CsA. In addition, the VEGF effect was significantly attenuated by siRNA against GATA2 (Figure 6C). Similar results were observed for tumor cell-conditioned medium (Figure 6D). Together with the real-time PCR findings, these findings support an essential role for GATA2 and NFATc1, -c2, and -c3 transcription factors in mediating inducible DSCR-1s expression.

NFATc and GATA2 regulate inducible expression of DSCR-1s in vivo. To determine the role of NFAT in mediating DSCR-1s expression in vivo, we pretreated DSCR-1s-lacZ-Hprt mice with CsA (1 mg/kg i.p. every second day for 2 weeks) prior to VEGF administration. CsA significantly inhibited VEGF induction of lacZ (Figure 7, A and B, shows aorta and lung; Supplemental Figure 6, A and B, shows diaphragm and chest wall). In B16-F1 melanoma xenografts, injection with $1 \mathrm{mg} / \mathrm{kg}$ CsA every other day for 20 days resulted in a profound reduction in lacZ staining (Figure $7 \mathrm{C}$ ). To elucidate the role of GATA2 in mediating inducible expression of DSCR-1s in vivo, we employed a microRNA against GATA2 (miGATA2). Treatment of cultured mouse endothelial cells (MS-1 cells) with miGATA2, but not miControl, significantly (89\%) inhibited expression of GATA2 (Supplemental Figure 7A). Systemically administrated miGATA2 resulted in marked downregulation of GATA2 protein expression in aortic endothelium (Supplemental Figure 7B) and GATA2 mRNA expression in aorta and lung (Supplemental Figure 7C). Importantly, miRNA against GATA2 significantly reduced VEGF-mediated induction of lacZ expression in the diaphragm, aorta, chest wall, and lung (Figure 7, D and E, shows aorta and lung; Supplemental Figure 6, C and D, shows diaphragm and chest wall). To determine the effect of miGATA2 on DSCR1s promoter activity in tumor endothelium, B16 melanoma cells were implanted subcutaneously in mice in the presence of adenovirus expressing either miGATA2 or miControl (Ad-miGATA2 or 
A

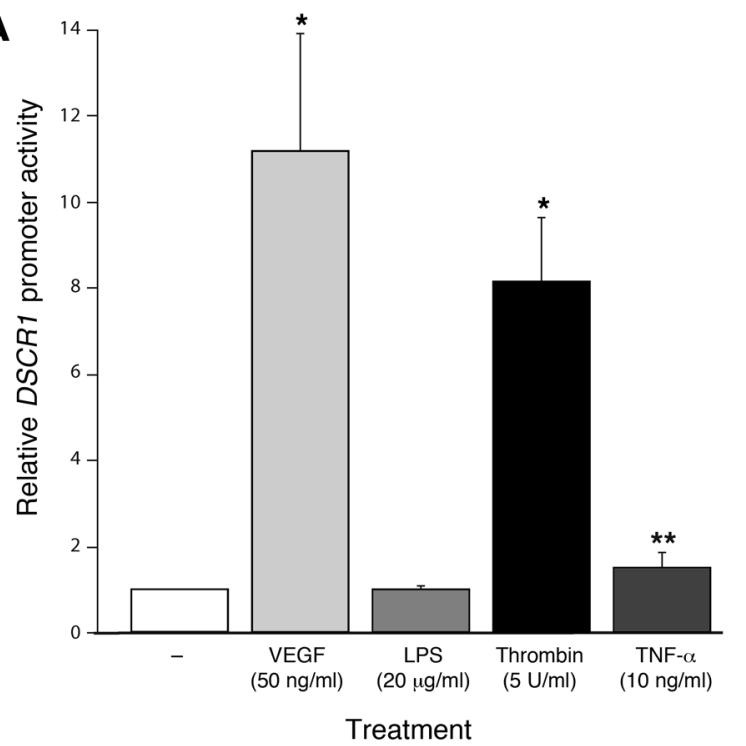

C

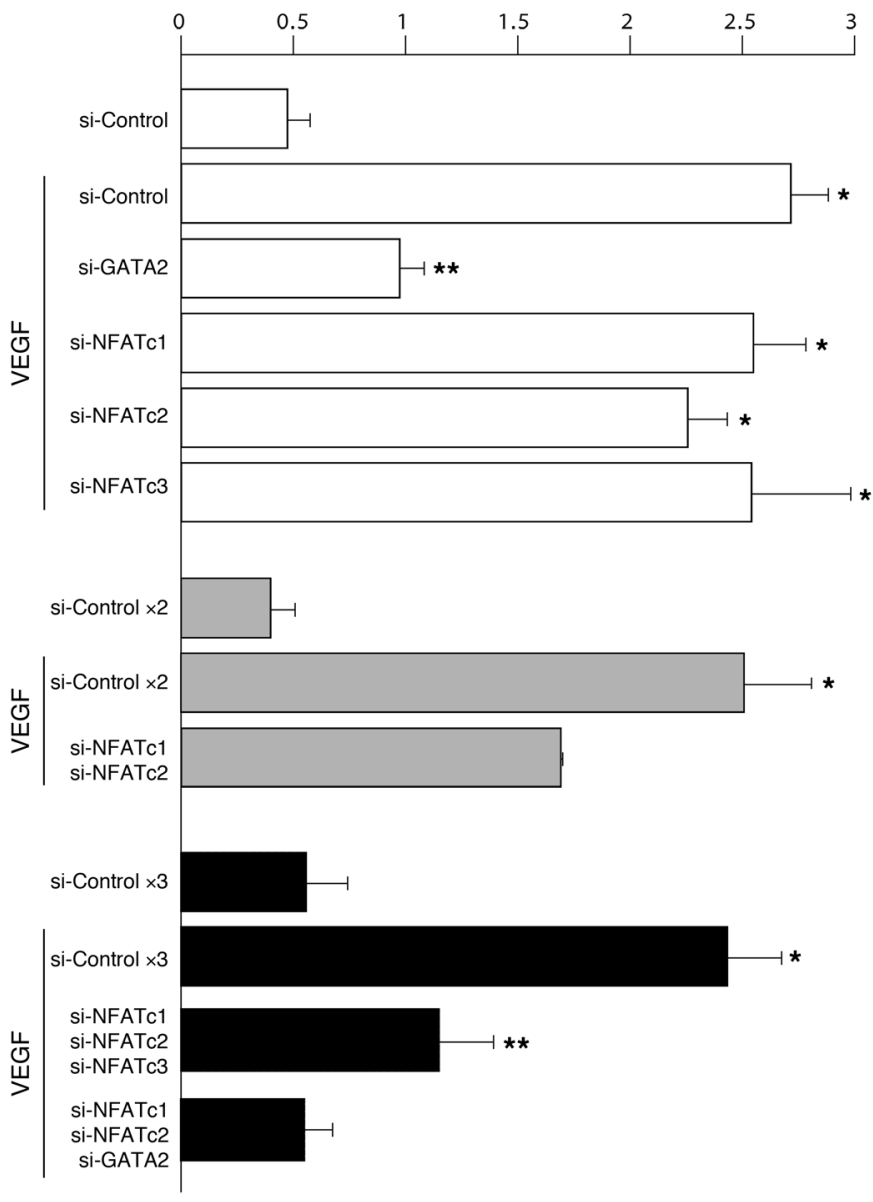

B

DSCR-1 mRNA expression

relative to cyclophilin $\mathrm{A}$

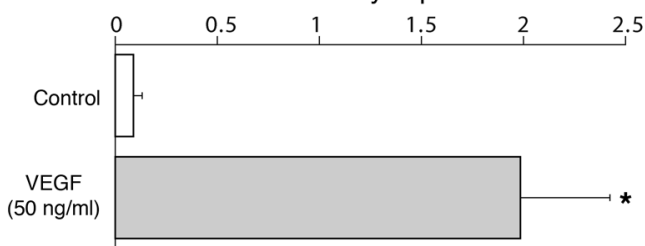

LPS
$(20 \mu \mathrm{g} / \mathrm{ml})$

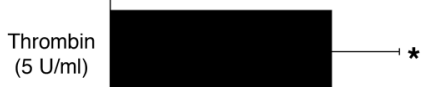

TNF- $\alpha$

(10 ng/ml)

\section{Figure 5}

GATA- and NFATc-dependent DSCR-1s expression in cultured microvascular endothelial cells. (A) HMVECs were transiently transfected with DSCR-1-luc2cp; serum starved for 16 hours; incubated for 2 hours with VEGF, LPS, thrombin, or TNF- $\alpha$; and then assayed for luciferase activity. The results show the mean \pm standard deviation of luciferase light units (relative to untreated cells) obtained in triplicate from at least 3 independent experiments. ${ }^{\star} P<0.001,{ }^{\star \star} P<0.05$ compared with untreated control. (B) Confluent HMVECs were serum-starved for 16 hours, then treated with VEGF, LPS, thrombin, or TNF- $\alpha$ for 1 hour. Total RNA was harvested and assayed by quantitative real-time PCR using DSCR-1s-specific primers. The results show the mean \pm standard deviation of expression levels relative to cyclophilin A obtained in 5 independent experiments. ${ }^{*} P<0.01$ compared with untreated control. (C) HMVECs were transfected with $40 \mathrm{nM}$ control siRNA or siRNA against GATA2 and NFATc1, -c2, and -c3 alone or in combination. Transfected cells were treated in the absence or presence of VEGF and assayed for DSCR-1 mRNA by real-time PCR. The results show the mean \pm standard deviation of expression levels relative to cyclophilin A obtained in triplicate from 3 independent experiments. ${ }^{*} P<0.001$, ${ }^{* *} P<0.05$ compared with si-Control without VEGF in each experiment. $\times 2$ and $\times 3,2$-fold molar $(80 \mathrm{nM})$ and 3 -fold molar (120 nM) siRNA treatments, respectively.
Ad-miControl). After 2 weeks, the mice received intratumor injection of $1 \times 10^{10} \mathrm{PFU}$ of Ad-miGATA 2 or miControl. Three days later, xenografts were harvested, sectioned, and stained for lacZ. These studies show that DSCR1s promoter activity in tumor vessels was abolished in the presence of miRNA against GATA2 (Figure 7F).
Together, these data strongly suggest a role for NFATc and GATA2 in the induction of DSCR-1s expression in the endothelium.

DSCR-1s attenuates endothelial cell activation in vivo. We previously demonstrated that DSCR1s attenuates VEGF-mediated activation of cultured endothelial cells (10). Together with our present find- 
A
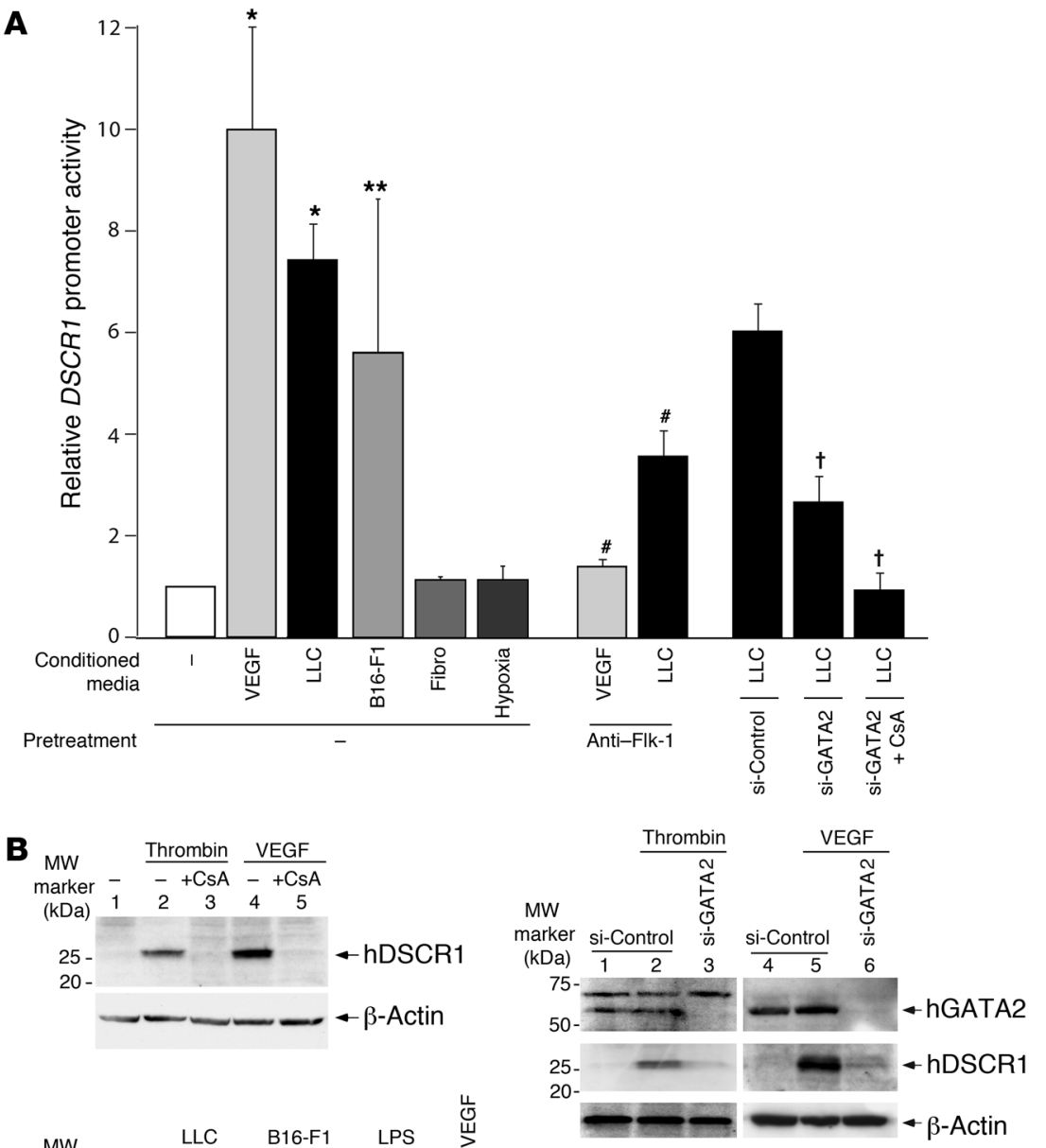

Figure 6

Modulatable DSCR1s promoter activity and protein expression in cultured microvascular endothelial cells. (A) HMVECs were transiently transfected with DSCR-1-luc2cp. Cells were then transfected with siRNA or treated with anti-Flk-1 neutralizing antibody or CsA and subsequently exposed to conditioned medium. The results show the mean \pm standard deviation of luciferase light units (relative to untreated cells) obtained in triplicate from 3 independent experiments. ${ }^{*} P<0.001$, ${ }^{* \star} P<0.01$ compared with control; ${ }^{\dagger} P<0.001 \mathrm{com}$ pared with si-Control plus LLC-conditioned medium. ${ }^{\#} P<0.001$ compared with no-pretreatment controls. (B) Top left: HMVECs were pretreated with vehicle control (lanes 1, 2, and 4) or $1 \mu \mathrm{M} \mathrm{CsA}$ (lanes 3 and 5) and then incubated in the absence (lane 1) or presence of thrombin (lanes 2 and 3) or VEGF (lanes 4 and 5). Cell lysates were subjected to immunoblotting with DSCR-1 antibody. Top right: HMVECs were transfected with control siRNA (lanes 1, 2, 4, and 5) or GATA2 siRNA (lanes 3 and 6 ) and then treated without (lanes 1 and 4) or with thrombin (lanes 2 and 3) or VEGF (lanes 5 and 6). Cell lysates were subjected to immunoblotting with DSCR-1 and GATA2 antibodies. Bottom: HMVECs were pretreated with vehicle control (lanes 1, 2, 4, 6 , and 8 ) or $1 \mu \mathrm{M} \mathrm{CsA}$ (lanes 3, 5, and 7) and then incubated in the absence (lane 1) or presence of conditioned medium from LLC (lanes 2 and 3 ), B16-F1 (lanes 4 and 5), LPS (lanes 6 and 7), or VEGF (lane 8). Membranes were stripped and reprobed with anti- $\beta$-actin antibody.

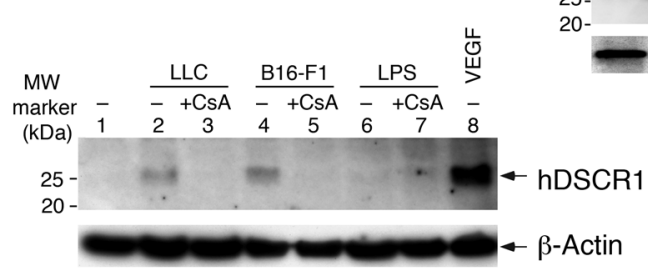

ings, these data led us to hypothesize that VEGF- and LPS-inducible expression of DSCR-1s in mice may serve as a negative feedback inhibitor of endothelial activation in vivo. To test this hypothesis, we examined the effect of DSCR-1 deficiency or overexpression on endotoxemia phenotype. The generation of $D s \mathrm{cr}^{-/-}$mice, which carry a targeted deletion of both DSCR-1s and DSCR-1L, has been previously described (11). To overexpress DSCR-1s, we employed an adenoviral delivery system in which the endothelium-specific Flt1 promoter is coupled to DSCR-1s followed by an IRES-EGFP cassette (Ad-Flt1-DSCR-1s). As evidence of its cell type specificity, Ad-Flt1-DSCR-1s resulted in detectable EGFP signal and DSCR-1s protein expression in HMVECs but not in B16-F1 melanoma cells (Supplemental Figure 8, A and B).

In the next series of experiments, mice were injected intravenously with Ad-Flt1-DSCR-1s or control adenovirus. Seven days later, organs were harvested and assayed for DSCR-1s and EGFP expression by real-time PCR and immunofluorescence microscopy, respectively. Ad-Flt1-DSCR-1s resulted in significantly increased DSCR-1s mRNA expression in the heart (278-fold), lung (953-fold), and liver (264-fold) (Supplemental Figure 8C). Immunostaining revealed colocalization of EGFP and PECAM-1 in endothelial cells of multiple organs (Supplemental Figure 8D, and data not shown). Thus, in vivo delivery of Ad-Flt1-DSCR-1s results in overexpression of DSCR-1s in the intact endothelium of mice.

Endotoxemia in mice is associated with a reduction in heart rate, blood pressure, and body temperature and an increase in circulating IL-6 levels $(12,13)$. This effect was accentuated in $D s \mathrm{cr}^{-/-}$mice (Supplemental Figure 9) and attenuated in DSCR-1s-overexpressing animals (Supplemental Figure 9). Although DSCR-1 deficiency has been linked to cardiac hypertrophy $(14,15)$, there was no gross evidence of cardiac abnormalities in control or LPS-treated mice (data not shown).

We previously reported that endotoxemia in mice is associated with increased circulating levels of $\operatorname{VEGF}(16,17)$. Further, we demonstrated that VEGF plays a pathogenic role in sepsis (17). Interestingly, resting levels of plasma VEGF were 5.0-fold higher in $D s c r 1^{-/-}$mice compared with wild-type littermates (Figure $8 \mathrm{~A})$. In response to LPS treatment, $\mathrm{Dscr1^{-/ }}$ mice demonstrated super-induction of circulating VEGF levels (2.2-fold higher vs. wild-type mice) (Figure 8A). Ad-Flt1-DSCR-1s-injected mice had no change in resting VEGF levels. However, in response to endotoxemia, DSCR-1s-overexpressing mice demonstrated a 


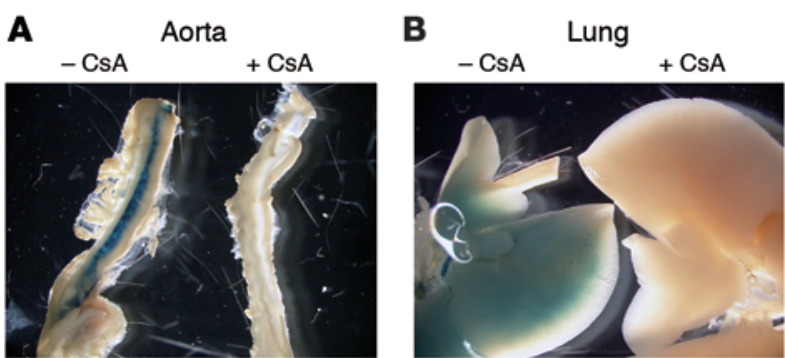

C

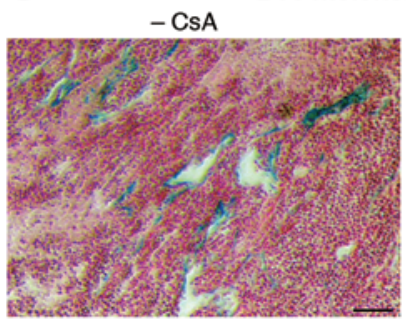

D Aorta

Ad-miControl Ad-miGATA2

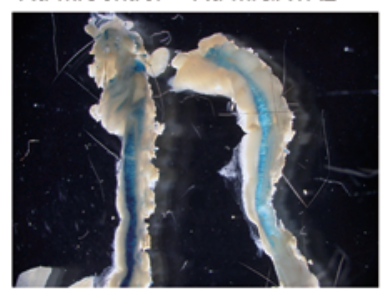

$\mathbf{F}$

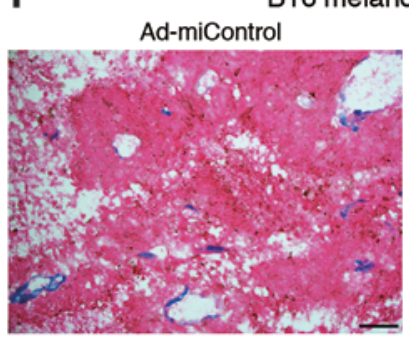

E

Ad-miControl Ad-miGATA2
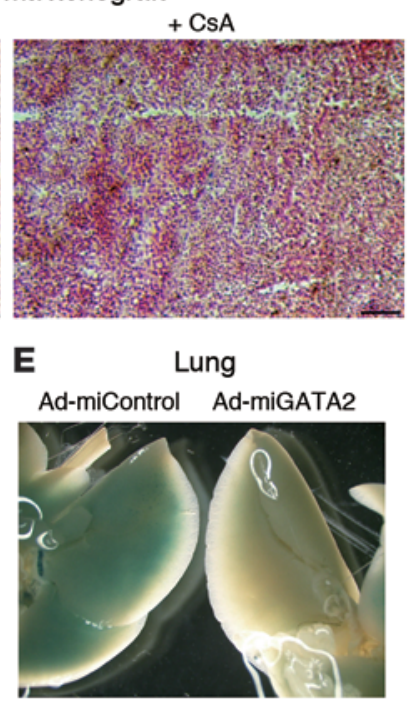

Lung

noma xenograft

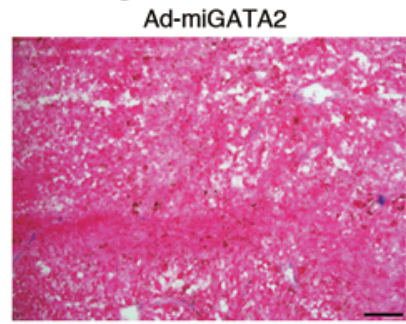

Figure 7

Administration of CsA and Ad-miGATA2 inhibits DSCR-1 promoter activation in Hprt transgenic mice. (A-C and D-F) Whole-mount lacZ staining of aorta and lung from VEGF-injected mice pretreated with or without CsA. (C) lacZ staining of B16-F1 melanoma xenografts from DSCR-1-lacZ-Hprt mice treated with or without $1 \mathrm{mg} / \mathrm{kg} \mathrm{CsA}$ i.p. every second day. Scale bars: $50 \mu \mathrm{m}$. (D-F) Same protocol as in A-C, except that mice were administered $1 \times 10^{10} \mathrm{PFU}$ of Ad-miControl or Ad-miGATA2 i.v. on days 0,2 , and 7 instead of receiving CsA. The results are representative of 4 (for $\mathrm{CsA}$ ) and 3 (for Ad-miGATA2) independent experiments. Scale bars: $50 \mu \mathrm{m}$.

$61 \%$ reduction in circulating VEGF levels compared with septic Ad-Flt1-control (Figure 8A).

To assay for endothelial activation, we employed real-time PCR to measure mRNA expression of E-selectin, ICAM-1, and VCAM-1 in tissues from mice 6 hours after injection of PBS (control) or LPS. Compared with wild-type littermate controls, LPS-treated Dscr1 $1^{-/-}$mice demonstrated super-induction of E-selectin in the heart and lung, ICAM- 1 in heart, and VCAM- 1 in lung. In contrast,

LPS-mediated induction of cell adhesion molecules was attenuated by overexpression of DSCR-1s (Figure 8B).

Next, we employed Mac1 staining to assay for leukocyte infiltration in the heart, lung, and liver. As shown in Figure 9, DSCR-1-null mutation resulted in increased numbers of activated leukocytes in the heart and lung (2.7- and 2.0-fold, respectively) compared with wild-type littermates. No such changes were observed in the liver, where inflammatory mediators failed to upregulate endogenous Dscr1s (see Figure 3B). In contrast to the effect of DSCR-1 knockout, overexpression of DSCR-1s resulted in a marked reduction in leukocyte infiltration in the heart, lung, and liver.

We have recently shown that hyperactivation of the VEGF/calcineurin/NFAT pathway triggers apoptosis in DSCR-1-deficient tumor endothelial cells (18). Given that $D s c r 1^{-/-}$mice have elevated circulating levels of VEGF (Figure 8A), we hypothesized that endotoxemia may result in increased endothelial cell apoptosis in $\mathrm{Dscr1} 1^{-/-}$ mice. To test this hypothesis, we carried out TUNEL assay in tissue sections from the heart and lung of LPS-treated DSCR-1-null mice and their wild-type littermates. As shown in Figure 10A, endotoxemic wild-type mice demonstrated a small number of TUNELpositive endothelial cells in the heart and even fewer in the lung. However, in $D s \mathrm{cr}^{-/-}$mice, LPS administration resulted in a marked increase in the number of TUNEL-positive cells in both organs.

Finally, in survival studies, LPS-treated $D s \mathrm{cr}^{-/-}$mice demonstrated markedly increased mortality compared with endotoxemic wild-type littermates (Figure 10B, left). In contrast, Ad-mediated overexpression of DSCR-1s conferred a survival advantage compared with Ad-Flt1-control (Figure 10B, right).

\section{Discussion}

In the present study, we show that expression of the DSCR-1s isoform is temporally and spatially regulated in the intact endothelium, and we provide new evidence for a critical role of DSCR-1s in the host response. The intergenic DSCR1s promoter exhibited robust and uniform endothelial expression during the critical embryonic stages of vasculogenesis and angiogenesis. However, the DSCR1s promoter was markedly downregulated after E14 and in the adult exhibited only low-level, vascular bed-restricted activity in the heart, brain, lung, and kidney. These findings suggest that the DSCR-1s isoform plays a particularly important role during embryonic development. In contrast to DSCR-1s, the DSCR-1L isoform is preferentially expressed in the fetal brain and adult heart of humans and rats (19) and in the brain of adult mice (see Figure 3B). These differences in expression between DSCR-1s and DSCR-1L may indicate that the two isoforms have unique and differing functions.

We and others have previously reported that VEGF induces expression of DSCR-1s in cultured endothelial cells $(8,20)$. Here, we have extended these results by showing that systemically administered VEGF stimulates DSCR-1s expression and promoter activity in selected vascular beds in vivo, including the heart, brain, lung, and kidney. In contrast, DSCR-1L expression was unchanged. Vascular bed-specific induction of DSCR-1s did not simply reflect local differences in VEGF signaling. Indeed, VEGF administration resulted in phosphorylation of the VEGF receptor Flk-1 in otherwise DSCR-1s-negative organs such as the liver. These findings suggest that differential expression of DSCR-1s in response to VEGF is mediated at a post-receptor level.

The systemic administration of LPS also induced organ-specific expression of DSCR-1s. However LPS, compared with VEGF, treat- 
A

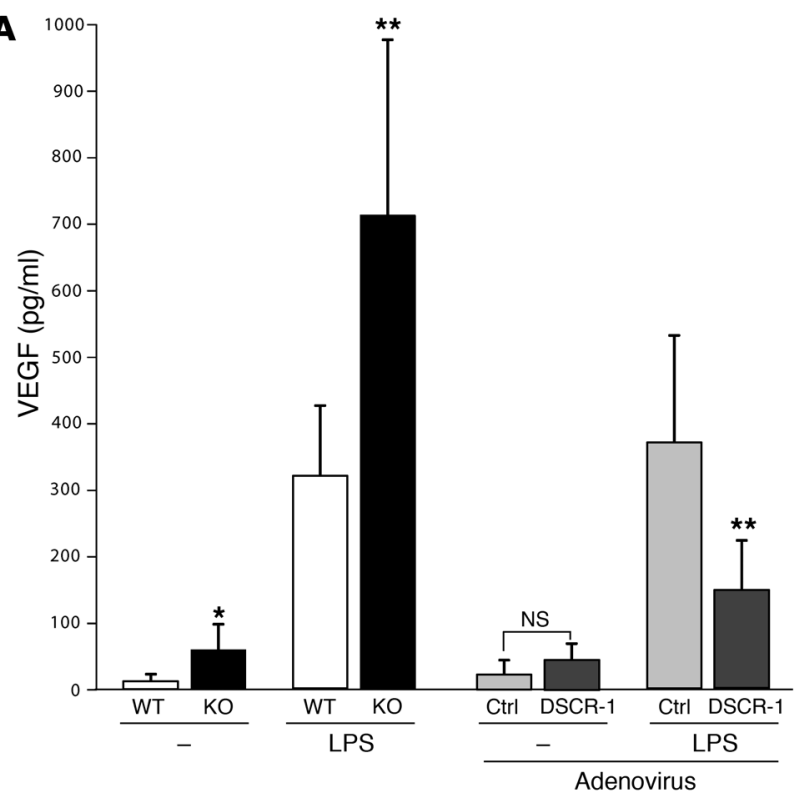

\section{Figure 8}

DSCR-1 levels influence inflammatory marker expression in sepsis. Wild-type mice, Dscr1-l- mice (KO), or mice injected with Ad-Flt1-control (Ctrl) or Ad-Flt1-DSCR-1 (DSCR-1) were injected i.p. with or without $16 \mathrm{mg} / \mathrm{kg}$ LPS for 20 hours. (A) Blood was harvested for plasma, and VEGF levels were measured using ELISA. Data are expressed as mean \pm standard deviation; $n=10 .{ }^{*} P<0.001,{ }^{* \star} P<0.01$ compared with wild-type or Ad-Flt1-control. (B) Heart and lung were harvested and assayed by real-time PCR for E-selectin, ICAM-1, and VCAM-1 mRNA expression. Data are expressed as mean \pm standard deviation; $n=4$. ${ }^{\dagger} P<0.001, \ddagger P<0.01, \S P<0.05$ compared with wild-type or Ad-Flt1-control injected with LPS.

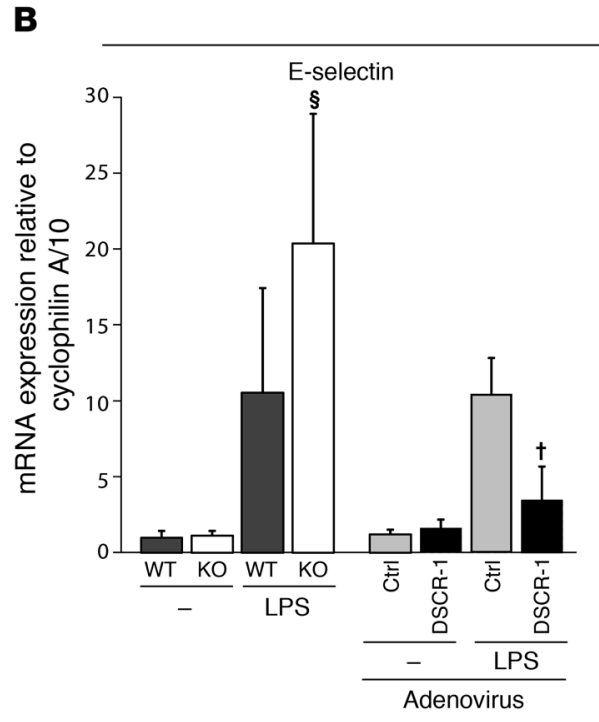

Heart
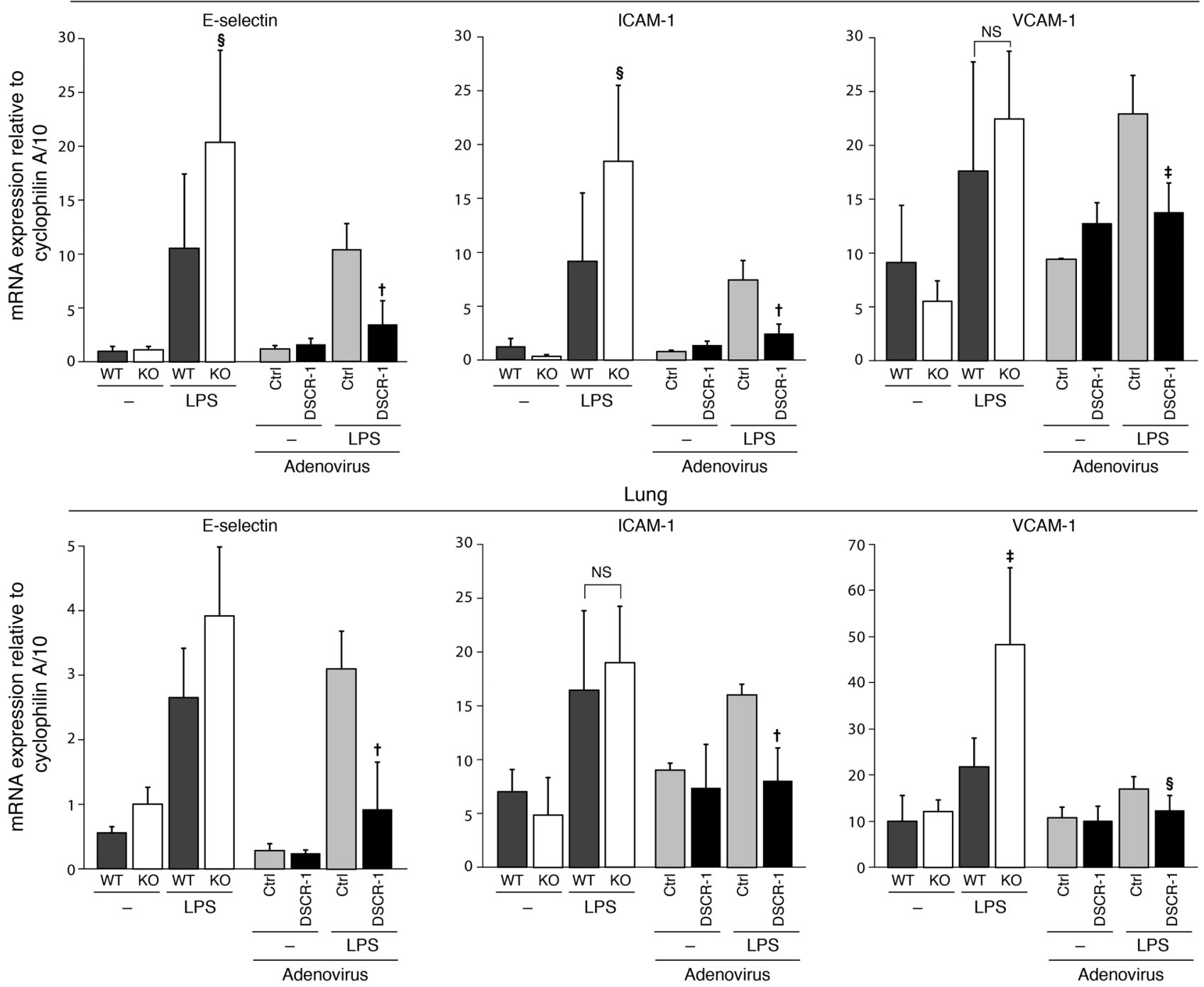

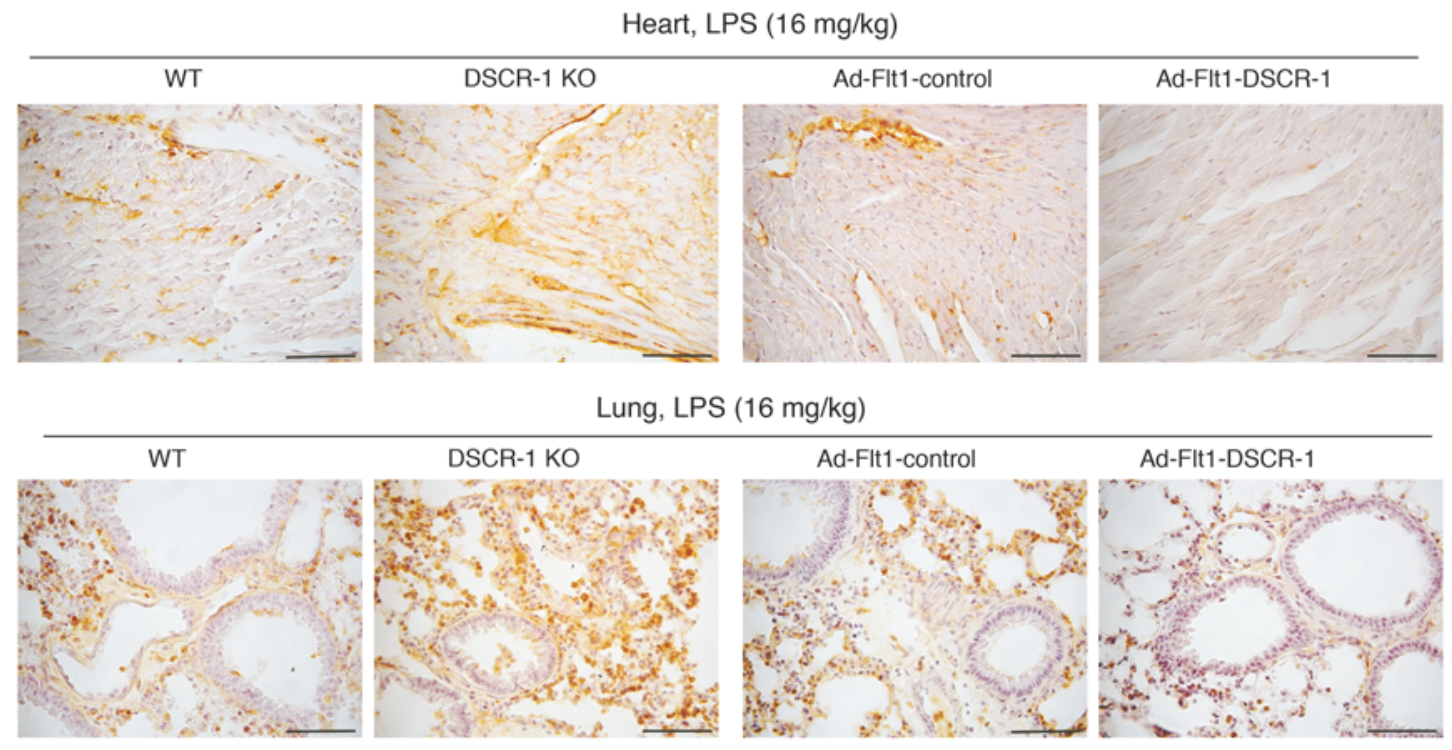

Liver, LPS (16 mg/kg)
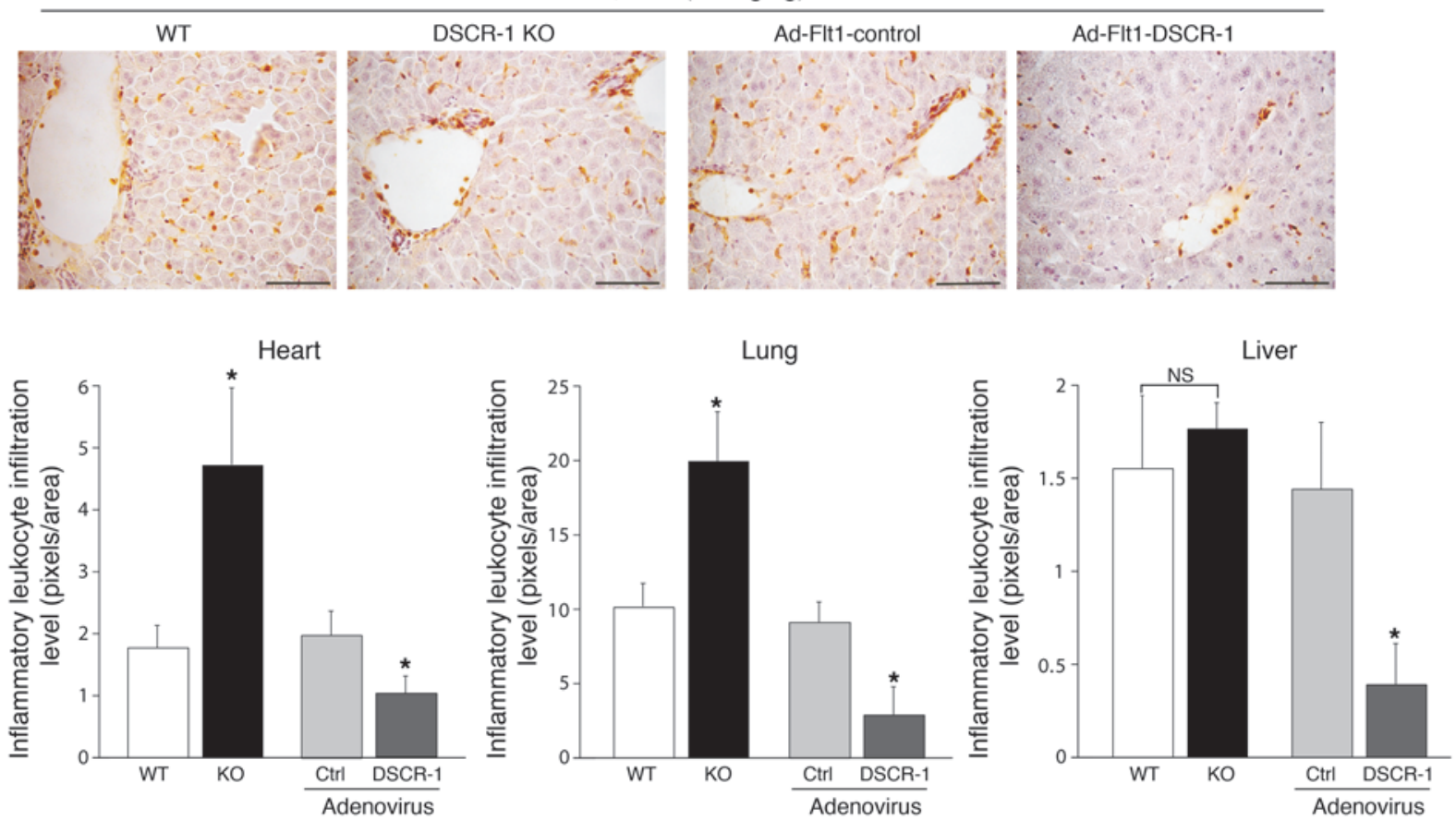

\section{Figure 9}

DSCR-1 levels influence inflammatory leukocyte infiltration rate in sepsis. Heart, lung, and liver of wild-type mice, Dscr1 $1^{-1-}$ mice, or mice injected with Ad-Flt1-control or Ad-Flt1-DSCR-1 were harvested, cryosectioned, and stained with anti-Mac1 antibody. Mac1-positive activated leukocytes (brown) were quantified based on the analysis of 6 independent sections (bar graphs). Data are expressed as mean \pm standard deviation. ${ }^{*} P<0.01$, compared with wild-type or Ad-Flt1-control injected with LPS. Scale bars: $50 \mu \mathrm{m}$.

ment of cultured endothelial cells had little effect on DSCR-1s expression. This discordance suggests that endotoxemia-associated induction of DSCR-1s results not from direct LPS-mediated Tolllike receptor 4 signaling in endothelial cells, but rather from secondary effects of LPS. For example, endotoxemia is associated with elevated levels of VEGF and thrombin, both of which have been implicated in sepsis pathophysiology $(17,21)$ and shown to induce expression of DSCR-1s in endothelial cells $(8,10)$. Further studies are needed to determine the molecular basis for vascular bed-spe- cific regulation of DSCR-1s expression under both basal and inducible conditions. The DSCR-1s-lacZ mice generated in this study should serve as a useful tool for dissecting these mechanisms.

Using siRNA or adenoviral miRNA approaches to downregulate transcription factor expression in endothelial cells and mice, respectively, we have shown that the DSCR1s promoter is activated via NFATc1, -c2, and -c3 and GATA2. There is evidence that DSCR-1L, which lacks NFAT consensus elements, is under the control of the Notch pathway. For example, during keratinocyte differen- 
A

WT
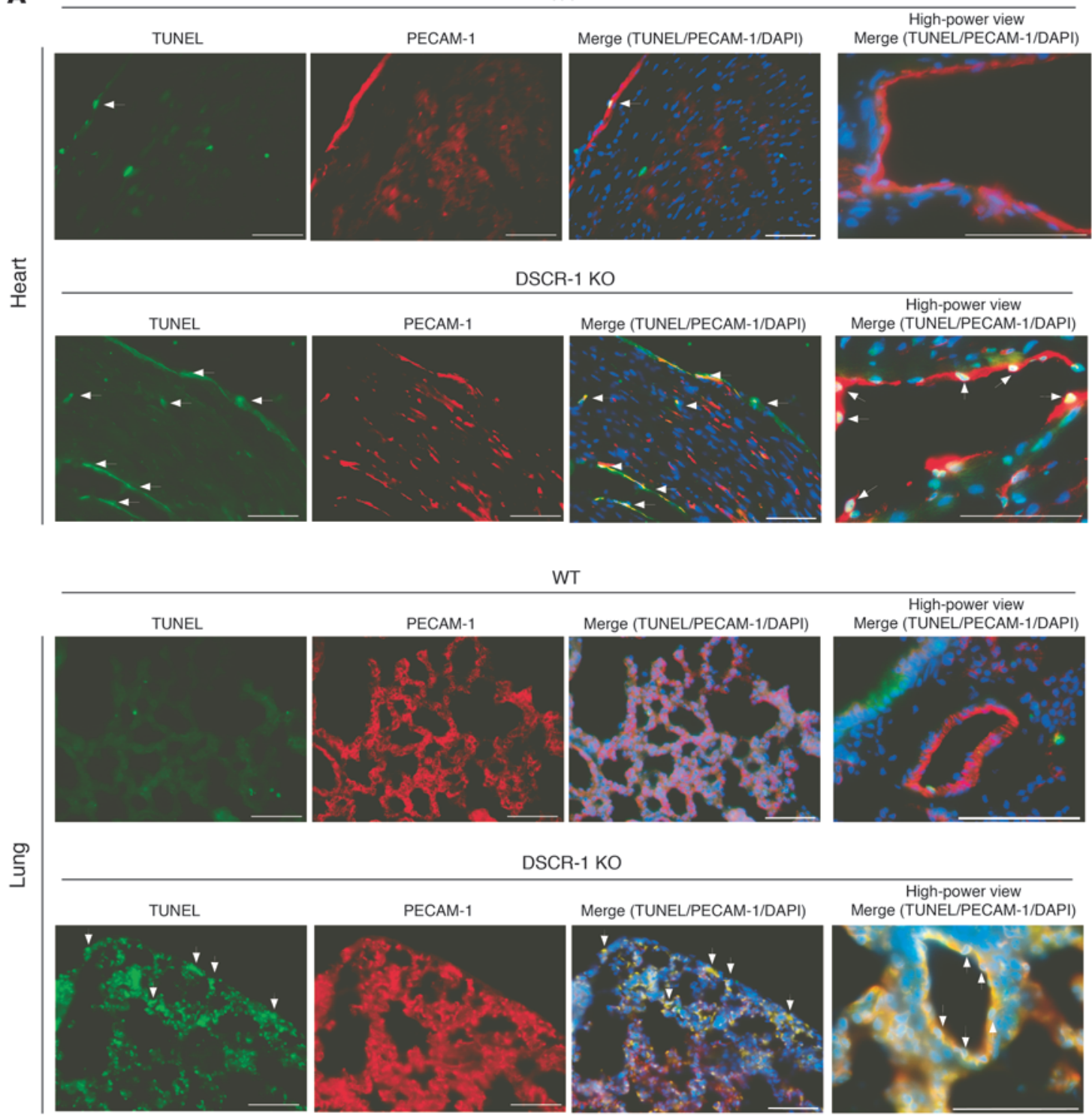

B

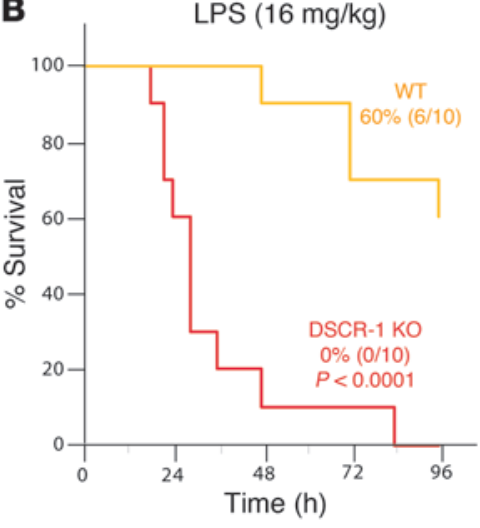

LPS

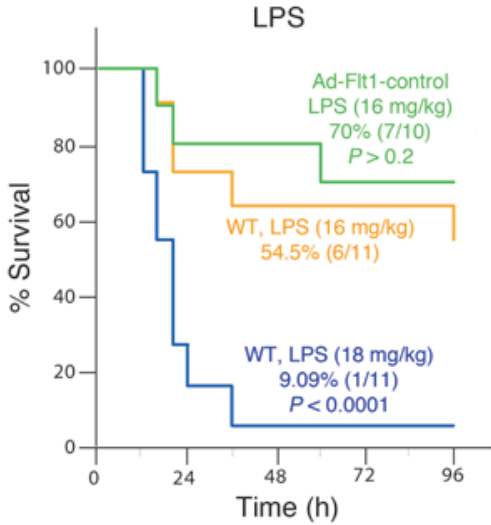

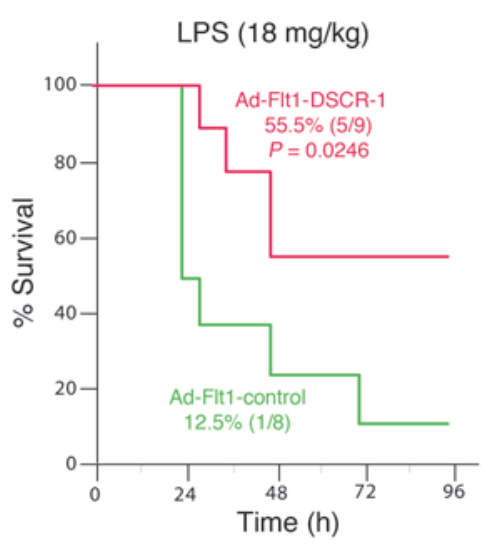

Figure 10

DSCR-1 modulates endothelial cell survival and sepsis mortality. (A) LPS-treated heart and lung were harvested and processed for TUNEL (green), PECAM-1 (red), or DAPI (blue) staining. Arrows indicate TUNEL-reactive apoptotic cell nuclei. Scale bars: 50 um. (B) Survival studies were carried out in $\mathrm{Dscr}^{-/-}(\mathrm{KO})$ or wild-type littermates administered $16 \mathrm{mg} / \mathrm{kg}$ LPS i.p. (left panel). Alternatively, mice were administered $5 \times 10^{9} \mathrm{PFU}$ of Ad-Flt1-control or Ad-Flt1-DSCR-1 i.v. and 3 days later received 16 or $18 \mathrm{mg} / \mathrm{kg}$ LPS i.p. (middle and right panels). Percentages of mice (surviving/total) are indicated. 
tiation, increased Notch activity was shown to downregulate DSCR-1L by promoting binding of Hes- 1 to the exon 1 promoter (7). In non-endothelial cells, DSCR-1L functions like DSCR-1s in inhibiting calcineurin (22-24). Indeed, in keratinocytes, Notch $1 /$ Hes-1-mediated reduction of DSCR-1L levels led to an accentuation of calcineurin/NFAT signaling, which in turn is predicted to activate the DSCR1s promoter. It is interesting to speculate that a similar mechanism of gene regulation is operative in endothelial cells. However, a recent study demonstrated that DSCR-1L does not inhibit, but rather activates calcineurin/NFAT in endothelial cells and promotes angiogenesis (25). Clearly, further studies are required to delineate the regulatory mechanisms and functions of DSCR-1s and DSCR-1L in the endothelium.

As an endogenous inhibitor of calcineurin, DSCR-1s acts in a negative feedback regulatory loop to attenuate endothelial cell activation. For example, DSCR-1s has been shown to suppress VEGF induction of E-selectin, VCAM-1, tissue factor, and COX-2 in cultured endothelial cells (20). Conversely, siRNA-mediated knockdown of DSCR-1 accentuated VEGF stimulation of tissue factor, E-selectin, and VCAM-1, while knockdown of NFATc1 blocked gene expression (20). In other studies, DSCR-1s overexpression in HUVECs inhibited VEGF or thrombin induction of tissue factor, IL-8, VCAM-1, E-selectin, monocyte chemoattractant protein-1 (MCP-1), and ICAM-1 $(8,10)$. To determine whether DSCR-1s plays an antiinflammatory role in vivo, we employed an endotoxemia model in DSCR-1-null mice and in mice overexpressing DSCR-1s. Because endotoxemia stimulates DSCR-1s expression primarily in the endothelium, we used the human Flt1 promoter (spanning the region between -748 and +284 ) for adenovirus-mediated expression of DSCR-1s in vivo. This promoter has been shown to direct strong and uniform activity in the endothelium of Hprt locus-targeted mice (26). Moreover, the Flt1 promoter has been used successfully in an adenoviral system to direct expression in lung endothelium (27). Consistent with these data, we observed strong expression of DSCR-1s and EGFP in the lung of Ad-Flt1-DSCR-1s-injected mice.

Importantly, we have demonstrated reciprocal effects of DSCR-1 deficiency and DSCR-1s overexpression on sepsis morbidity/mortality. Our data suggest that DSCR-1s protects against LPS-mediated bradycardia, hypotension, and hypothermia; attenuates circulating levels of inflammatory mediators and mRNA expression of endothelial activation markers; and inhibits leukocyte infiltration in the heart, lung, and liver. Together with our findings in cultured endothelial cells $(8,10)$, these in vivo data suggest that DSCR-1s is protective in sepsis, in part by inhibiting NFAT-mediated endothelial cell activation and secondary inflammation. Interestingly, DSCR-1 deficiency was associated with increased circulating levels of VEGF. Given that VEGF activates NFAT, among other signaling pathways, and has been shown to play a pathophysiological role in sepsis (17), this effect may further contribute to the increased sepsis morbidity and mortality in $D s \mathrm{cr}^{-/-}$mice.

We have previously shown that the overexpression of DSCR-1s inhibited neovascularization in Matrigel plugs and xenografts in mice (8). In contrast to these findings, $D s \mathrm{cr}^{-/-}$mice demonstrated reduced blood vessel formation in Matrigel, corneal micropocket, and tumor xenograft assays (18). Dscr $1^{-1-}$ endothelial cells displayed hyperactivation of the calcineurin/NFAT pathway and increased sensitivity to VEGF signaling (18). However, rather than inducing cell proliferation, VEGF-mediated activation of calcineurin/NFAT in Dscr1-/- endothelial cells "re-routed" downstream sig- naling, resulting in increased apoptosis, which thus explains the paradoxical reduction in neovascularization (18). Consistent with these findings, we found that endotoxemia promoted endothelial apoptosis in Dscr1 $1^{-/}$mice. Thus, DSCR-1s plays a dual role in the host response to infection by protecting against excessive endothelial activation and promoting endothelial survival. Based on these data, we propose that DSCR-1s represents a novel therapeutic target in inflammatory diseases.

\section{Methods}

Generation and analysis of Hprt-targeted transgenic mice. Hprt-targeted ES cells containing the DSCR-1-lacZ transgene were used to generate chimeric mice as previously described (26). Chimeric males were bred to C57BL/6J females to obtain agouti offspring. Female agouti offspring were then bred to wild-type males to generate hemizygous male mice. Analysis of embryos and hemizygous adult male tissues was carried out as previously described $(26,28)$. The level and pattern of transgene expression were compared with those of the littermate negative control (same genetic background) mice. All animal studies were approved by the Institutional Animal Care and Use Committees at the University of Tokyo and Beth Israel Deaconess Medical Center.

Materials and cell culture. Human and murine VEGF were obtained from R\&D Systems. Thrombin and CsA were obtained from Calbiochem. LPS and polyinosinic acid potassium salt [poly(I)] were from Sigma-Aldrich. Antibodies against PECAM-1, VCAM-1, E-selectin, and Mac1 were obtained from BD Biosciences - Pharmingen. Goat anti-GATA2 antibody was from R\&D Systems. Rabbit anti-EGFP and anti-lacZ antibodies were from Medical $\&$ Biological Laboratories Co. Mouse monoclonal anti-GATA2 and DSCR-1 antibodies were generated with baculovirus glycoprotein 64-GATA2 and baculovirus glycoprotein 64-DSCR1 fusion proteins, respectively, as the antigen. HMVECs (Clonetics) were grown in Endothelial Growth Medium2-MV (EGM-2-MV) Bullet Kit (Clonetics). HMVECs were used within the first 10 passages. Mouse B16-F1 melanoma (CRL-6323; ATCC), mouse LLC (CRL-1642; ATCC), human skin fibroblast (Clonetics), and mouse MS-1 (CRL-2279; ATCC) cells were grown in DMEM plus 10\% FBS.

Construction of plasmids and adenoviruses. The DSCR-1 intergenic promoter was cloned as described previously (8). To generate the Hprt targeting vector, the DSCR-1 promoter fragment $(-1,664 /+84)$ was subcloned into pSDK-lacZ, then ligated with pMp8II. For luciferase assays, DSCR-1 promoter was subcloned into pGL4.12 (luc2cp) (Promega). For construction of adenovirus expressing EGFP alone (control) or DSCR-1 plus EGFP driven by the Flt1 promoter, a 1,026-bp fragment of the human Flt1 promoter was inserted into $\mathrm{MfeI} / \mathrm{NheI}$-digested pShuttle (Clontech). The resulting vector was used to insert IRES2-EGFP or DSCR-1-IRES2-EGFP gene cassettes. The resulting plasmids were transferred to Adeno-X DNA (Clontech) using the Adeno-X adenoviral expression system (Clontech).

To generate the Ad-miGATA2, miRNA sequences (Supplemental Table 1) against mouse GATA2 were ligated into pcDNA6.2-GW/EmGFP-miR (Invitrogen), then transferred to $\mathrm{pAd} / \mathrm{CMV} / \mathrm{V} 5$-DEST gateway vector according to the manufacturer's instructions (Invitrogen).

Real-time PCR. RNA was extracted from endothelial cells using the TRIzOL reagent (Invitrogen). For harvesting the RNA from mouse organs, tissues were quickly soaked in RNAlater solution (Ambion), then immersed with TRIzoL and homogenized using a tissue grinder (Polytron). Two micrograms of total RNA were reverse transcribed using SuperScript III enzyme and oligo-dT primer as specified by Invitrogen. Real-time PCR including SYBR Green reagent was performed on an instrument according to instructions provided by the manufacturer (Applied Biosystems). Primer pairs are shown in Supplemental Table 1. Expression values are represented as mean \pm standard deviation relative to cyclophilin A expression. 
Transient transfection of endothelial cells with plasmid DNA and siRNA and analysis of luciferase activity. HMVECs were transfected with plasmid DNA using FuGENE HD reagent (Roche), and luciferase activities were calculated using the Dual-Luciferase assay kit (Promega) as previously described (29). The transfected cells were serum starved, preincubated for 30 minutes with $1 \mu \mathrm{M}$ CsA or anti-Flk-1/KDR neutralizing antibody (R\&D Systems), and then incubated for 2 hours with conditioned medium from confluent B16-F1 or LLC tumor cells cultured for 2 days in DMEM with $2 \%$ FBS or incubated with DMEM plus $2 \%$ FBS in the presence or absence of $50 \mathrm{ng} / \mathrm{ml}$ VEGF for 2 days. For siRNA transfection, HMVECs were incubated with a mixture of 40-120 nM Stealth siRNA (Invitrogen) and Lipofectamine Max (Invitrogen) for 24 hours. After serum starvation (EBM-2 plus 0.5\% FBS) for 16 hours, cells were treated with $50 \mathrm{ng} / \mathrm{ml}$ VEGF, $5 \mathrm{U} / \mathrm{ml}$ thrombin, $10 \mathrm{ng} / \mathrm{ml} \mathrm{TNF}-\alpha$, or $20 \mu \mathrm{g} / \mathrm{ml} \mathrm{LPS}$ for 2 hours and then processed for luciferase activity, real-time PCR, or Western blotting. Targeted siRNA sequences are shown in Supplemental Table 1.

Administration of CsA and GATA2 miRNA to mice. Matrigel (Clontech) containing $1 \times 10^{6} \mathrm{~B} 16-\mathrm{F} 1$ cells with vehicle or $20 \mu \mathrm{g}$ CsA was implanted s.c. into transgenic mice. The mice were subsequently treated with $1 \mathrm{mg} / \mathrm{kg}$ CsA i.p. every second day. After 20 days, tumor xenografts were harvested and processed for lac Z staining. Alternatively, mice were treated with CsA $(1 \mathrm{mg} / \mathrm{kg})$ every second day for 14 days and then injected with vehicle or VEGF. Organs were removed for whole-mount lacZ staining. In the case of miRNA treatment, Matrigel containing $1 \times 10^{6} \mathrm{~B} 16$-F1 cells and $1 \times 10^{10}$ PFU Ad-miGATA2 or Ad-miControl was implanted s.c. into mice. After 14 days, the same amounts of adenoviruses were injected into the tumor. After 3 days, xenografts were harvested and examined. Alternatively, mice were injected i.v. with $0.2 \mathrm{mg}$ poly(I) (30) and $1 \times 10^{10} \mathrm{PFU}$ of Ad-miGATA2 or Ad-miControl on days 0,2 , and 7. On day 7, the mice were injected with or without VEGF. Organs were removed for whole mount lacZ staining.

Immunohistochemistry. Mice were perfused with $2 \%$ paraformaldehyde in PBS. Tissues were then harvested and fixed with $2 \%$ paraformaldehyde for 2 hours and immersed in $30 \%$ sucrose for 20 hours at $4^{\circ} \mathrm{C}$. Cyrosectioned slides were treated with ice-cold acetone for 10 minutes and then processed for blocking with Protein Block Serum-Free (Dako) and incubation with first antibody diluted by Block Ace (in PBS) (Dainippon Sumitomo Pharma) for 20 hours at $4^{\circ} \mathrm{C}$. Alternatively, in the case of DSCR-1 immunohistology, sectioned slides were treated with HistoVT One solution (Nacalai Tesque) for 20 minutes at $70^{\circ} \mathrm{C}$, then washed with PBS and processed for antibody reaction. The dilution rates were: for PECAM-1 and GATA2 antibodies: 50 -fold; $\beta$-galactosidase antibody: 15,000-fold; EGFP antibody: 10,000-fold; and DSCR-1 antibody: $50 \mu \mathrm{g} / \mathrm{ml}$. Tissue plates were washed and incubated with an Alexa Fluorlabeled secondary antibody (Invitrogen) for 1 hour. The slides were then washed in PBS, mounted in ProLong Gold Anti-Fade Reagent with DAPI (Invitrogen), and examined by fluorescence microscopy. For staining of leukocytes, ice-cold acetone-fixed slides were pretreated with $3 \%$ $\mathrm{H}_{2} \mathrm{O}_{2}$ followed by Avidin/Biotin blocking reagent (Invitrogen) to block endogenous peroxidase and biotin. Subsequently, slides were processed for blocking (Dako) and incubation with anti-Mac1 antibody. To detect Mac1-positive cells, biotinylated anti-rat IgG and the VECTASTAIN Elite ABC kit (Vector Laboratories) was used according to the manufacturer's instructions. For detection of apoptotic cells in situ, VasoTACS In Situ Apoptosis detection kit was used according to the instructions provided by manufacturer (R\&D Systems). Briefly, cryosections were treated with Cytonin (R\&D Systems) for 30 minutes. The $3^{\prime}$ ends of cleaved DNA fragments were labeled with biotin-dUTP using the enzyme TdT. TUNEL-positive cells were detected with Alexa Fluor 488-conjugated streptavidin (Invitrogen). The same slides were coimmunostained with anti-PECAM-1 antibody and DAPI.

Survival studies. Survival studies were performed using endotoxemia models. Survival was assessed at 24, 48, 72, and 96 hours after $16-18 \mathrm{mg} /$ $\mathrm{kg}$ weight LPS injection. Male C57BL/6 mice at 8 weeks of age and male Dscr1 $1^{-/-}$mice and their age-matched wild-type littermates at 7-9 weeks of age mice were used in this study. In a study involving adenovirus administration, $5.0 \times 10^{9} \mathrm{PFU}$ of Ad-Flt1-hDSCR-1 or Ad-Flt1-control were intravenously injected 3 days prior to LPS administration.

Measurement of IL-6 and VEGF levels in plasma. Systemic blood samples were collected and centrifuged. Plasma was harvested and stored at $-80^{\circ} \mathrm{C}$ until all samples were prepared. Thawed plasma was diluted and the IL- 6 and VEGF level measured using Quantikine ELISA kit (R\&D Systems).

Statistics. Data are presented as mean \pm standard deviation or SEM as indicated. $P$ values were calculated using 2-tailed unpaired Student's $t$ test. The Wilcoxon log-rank test was used for mouse survival studies. Statistical tests and graphing were done with KaleidaGraph (Synergy Software). A $P$ value less than 0.05 was considered significant.

\section{Acknowledgments}

This study was supported by the Fund for Science and Technology of the Ministry of Education, Culture, Sports, Science and Technology-Japan (T. Minami); the Takeda Science Foundation, Japan (T. Minami); the Garrett B. Smith Foundation (S. Ryeom); the Smith Family Medical Foundation (S. Ryeom); and NIH grants HL082927 (to W.C. Aird) and HL076540 (to W.C. Aird).

Received for publication March 25, 2008, and accepted in revised form May 27, 2009.

Address correspondence to: Takashi Minami, Research Center for Advanced Science and Technology, The University of Tokyo, Tokyo 153-8904, Japan. Phone: 81-3-5452-5403; Fax: 81-3-54525232; E-mail: minami@med.rcast.u-tokyo.ac.jp. Or to: William C. Aird, Department of Molecular and Vascular Medicine, Beth Israel Deaconess Medical Center/Harvard Medical School, Boston, Massachusetts 02215, USA. Phone: (617) 667-1031; Fax: (617) 6671035; E-mail: waird@bidmc.harvard.edu.
1. Aird, W.C. 2007. Phenotypic heterogeneity of the endothelium. II. Representative vascular beds. Circ. Res. 100:174-190.

2. Aird, W.C. 2007. Phenotypic heterogeneity of the endothelium: I. Structure, function, and mechanisms. Circ. Res. 100:158-173.

3. Aird, W.C. 2006. Mechanisms of endothelial cell heterogeneity in health and disease. Circ. Res. 98:159-162.

4. Klee, C.B., Ren, H., and Wang, X. 1998. Regulation of the calmodulin-stimulated protein phosphatase, calcineurin. J. Biol. Chem. 273:13367-13370.

5. Fuentes, J.J., Pritchard, M.A., and Estivill, X. 1997. Genomic organization, alternative splicing, and expression patterns of the DSCR1 (Down syndrome candidate region 1) gene. Genomics. 44:358-361.

6. Yang, J., et al. 2000. Independent signals control expression of the calcineurin inhibitory proteins MCIP1 and MCIP2 in striated muscles. Circ. Res. 87:E61-E68.

7. Mammucari, C., et al. 2005. Integration of Notch 1 and calcineurin/NFAT signaling pathways in keratinocyte growth and differentiation control. Dev. Cell. 8:665-676.

8. Minami, T., et al. 2004. Vascular endothelial growth factor- and thrombin-induced termination factor, Down syndrome critical region-1, attenuates endothelial cell proliferation and angiogenesis.
J. Biol. Chem. 279:50537-50554.

9. Liu, L., Tsai, J.C., and Aird, W.C. 2000. Egr-1 gene is induced by the systemic administration of the vascular endothelial growth factor and the epidermal growth factor. Blood. 96:1772-1781.

10. Minami, T., Miura, M., Aird, W.C., and Kodama, T. 2006. Thrombin-induced autoinhibitory factor, Down syndrome critical region-1, attenuates NFAT-dependent vascular cell adhesion molecule-1 expression and inflammation in the endothelium. J. Biol. Chem. 281:20503-20520.

11. Ryeom, S., Greenwald, R.J., Sharpe, A.H., and McKeon, F. 2003. The threshold pattern of calcineurin-dependent gene expression is altered by 
loss of the endogenous inhibitor calcipressin. Nat. Immunol. 4:874-881.

12. Ci, X., et al. 2008. Ceftiofur regulates LPS-induced production of cytokines and improves LPS-induced survival rate in mice. Inflammation. 31:422-427.

13. Clark, N., et al. 2007. The transient receptor potential vanilloid 1 (TRPV1) receptor protects against the onset of sepsis after endotoxin. FASEB J. 21:3747-3755.

14. Vega, R.B., et al. 2003. Dual roles of modulatory calcineurin-interacting protein 1 in cardiac hypertrophy. Proc. Natl. Acad. Sci. U. S. A. 100:669-674.

15. Rothermel, B.A., et al. 2001. Myocyte-enriched calcineurin-interacting protein, MCIP1, inhibits cardiac hypertrophy in vivo. Proc. Natl. Acad. Sci. U. S. A. 98:3328-3333.

16. Yano, K., et al. 2008. Elevated levels of placental growth factor represent an adaptive host response in sepsis. J. Exp. Med. 205:2623-2631.

17. Yano, K., et al. 2006. Vascular endothelial growth factor is an important determinant of sepsis morbidity and mortality. J. Exp. Med. 203:1447-1458.

18. Ryeom, S., et al. 2008. Targeted deletion of the calcineurin inhibitor DSCR1 suppresses tumor growth. Cancer Cell. 13:420-431.

19. Fuentes, J.J., et al. 1995. A new human gene from the Down syndrome critical region encodes a proline-rich protein highly expressed in fetal brain and heart. Hum. Mol. Genet. 4:1935-1944.

20. Hesser, B.A., et al. 2004. Down syndrome critical region protein 1 (DSCR1), a novel VEGF target gene that regulates expression of inflammatory markers on activated endothelial cells. Blood. 104:149-158.

21. Kahn, M.L. 2008. Counteracting clotting in sepsis. Nat. Med. 14:918-919.

22. Fuentes, J.J., et al. 2000. DSCR1, overexpressed in Down syndrome, is an inhibitor of calcineurinmediated signaling pathways. Hum. Mol. Genet. 9:1681-1690.

23. Vega, R.B., Yang, J., Rothermel, B.A., Bassel-Duby, R., and Williams, R.S. 2002. Multiple domains of MCIP1 contribute to inhibition of calcineurin activity. J. Biol. Chem. 277:30401-30407.

24. Rothermel, B., et al. 2000. A protein encoded within the Down syndrome critical region is enriched in striated muscles and inhibits calcineurin signaling. J. Biol. Chem. 275:8719-8725.

25. Qin, L., et al. 2006. Down syndrome candidate region 1 isoform 1 mediates angiogenesis through the calcineurin-NFAT pathway. Mol. Cancer Res. 4:811-820.

26. Minami, T., Donovan, D.J., Tsai, J.C., Rosenberg, R.D., and Aird, W.C. 2002. Differential regulation of the von Willebrand factor and Flt-1 promoters in the endothelium of hypoxanthine phosphoribosyltransferase-targeted mice. Blood. 100:4019-4025.

27. Reynolds, P.N., et al. 2001. Combined transductional and transcriptional targeting improves the specificity of transgene expression in vivo. Nat. Biotechnol. 19:838-842.

28. Minami, T., et al. 2003. Ets motifs are necessary for endothelial cell-specific expression of a 723-bp Tie-2 promoter/enhancer in Hprt targeted transgenic mice. Arterioscler. Thromb. Vasc. Biol. 23:2041-2047.

29. Minami, T., and Aird, W.C. 2001. Thrombin stimulation of the vascular cell adhesion molecule- 1 promoter in endothelial cells is mediated by tandem nuclear factor-kappa B and GATA motifs. J. Biol. Chem. 276:47632-47641.

30. Haisma, H.J., et al. 2008. Polyinosinic acid enhances delivery of adenovirus vectors in vivo by preventing sequestration in liver macrophages. J. Gen. Virol. 89:1097-1105. 